\title{
Normal and Pathological NCAT Image and Phantom Data Based on Physiologically Realistic Left Ventricle Finite Element Models
}

5

Alexander I. Veress ${ }^{*}$, W. Paul Segars ${ }^{+}$, Jeffrey A. Weiss ${ }^{*}$, Benjamin M. W. Tsui ${ }^{+}$, and Grant T. Gullberg ${ }^{\#}$

10

* The University of Utah

Department of Bioengineering, and the

Scientific Computing and Imaging Institute

Salt Lake City, UT

15

+The Johns Hopkins University, Department of Radiology

Baltimore, MD

and

20

\#E. O. Lawrence Berkeley National Laboratory, Life Science Division

Berkeley, CA

$\underline{\text { Revision 2 }}$, IEEE Transactions on Medical Imaging

May 5, 2006 


\section{Abstract -}

The 4D NURBS-based Cardiac-Torso (NCAT) phantom, which provides a realistic model of the normal human anatomy and cardiac and respiratory motions, is used in medical imaging research to evaluate and improve imaging devices and techniques, especially

5 dynamic cardiac applications. One limitation of the phantom is that it lacks the ability to accurately simulate altered functions of the heart that result from cardiac pathologies such as coronary artery disease (CAD). The goal of this work was to enhance the 4D NCAT phantom by incorporating a physiologically based, finite-element (FE) mechanical model of the left ventricle (LV) to simulate both normal and abnormal cardiac motions. The geometry of the FE mechanical model was based on gated high-resolution x-ray multi-slice computed tomography (MSCT) data of a healthy male subject. The myocardial wall was represented as transversely isotropic hyperelastic material, with the fiber angle varying from -90 degrees at the epicardial surface, through 0 degrees at the mid-wall, to 90 degrees at the endocardial surface. A time varying elastance model was used to simulate fiber contraction, and

15 physiological intraventricular systolic pressure-time curves were applied to simulate the cardiac motion over the entire cardiac cycle. To demonstrate the ability of the FE mechanical model to accurately simulate the normal cardiac motion as well abnormal motions indicative of CAD, a normal case and two pathologic cases were simulated and analyzed. In the first pathologic model, a subendocardial anterior ischemic region was defined. A second model 20 was created with a transmural ischemic region defined in the same location. The FE based deformations were incorporated into the 4D NCAT cardiac model through the control points that define the cardiac structures in the phantom which were set to move according to the 
predictions of the mechanical model. A simulation study was performed using the FE-NCAT combination to investigate how the differences in contractile function between the subendocardial and transmural infarcts manifest themselves in myocardial SPECT images. The normal FE model produced strain distributions that were consistent with those reported in 5 the literature and a motion consistent with that defined in the normal 4D NCAT beating heart model based on tagged MRI data. The addition of a subendocardial ischemic region changed the average transmural circumferential strain from a contractile value of -0.19 to a tensile value of 0.03 . The addition of a transmural ischemic region changed average circumferential strain to a value of 0.16 , which is consistent with data reported in the literature. Model results demonstrated differences in contractile function between subendocardial and transmural infarcts and how these differences in function are documented in simulated myocardial SPECT images produced using the 4D NCAT phantom. In comparison to the original NCAT beating heart model, the FE mechanical model produced a more accurate simulation for the cardiac motion abnormalities. Such a model, when incorporated into the 4D NCAT phantom,

15 has great potential for use in cardiac imaging research. With its enhanced physiologicallybased cardiac model, the 4D NCAT phantom can be used to simulate realistic, predictive imaging data of a patient population with varying whole-body anatomy and with varying healthy and diseased states of the heart that will provide a known truth from which to evaluate and improve existing and emerging 4D imaging techniques used in the diagnosis of cardiac 20 disease. 
Index Terms - Left Ventricle, NCAT, SPECT phantom, ischemia, mechanical model, finite element, cardiac imaging research. 


\section{INTRODUCTION}

Diagnostic imaging techniques play a vital role in reducing the mortality rate and strain on the healthcare system caused by coronary artery disease (CAD) by providing more efficient methods to screen and manage cardiac patients. Left ventricular (LV) function is a

5 major diagnostic and prognostic indicator in patients with CAD [1-4]. Many non-invasive imaging modalities and tools are being studied and developed for diagnosing cardiac disease based on an analysis of the LV function. The goal of these studies is to develop methods that better detect, stratify, and monitor therapy for patients with CAD. As new imaging techniques and diagnostic methods emerge in response to cardiac disease, a major challenge is how to evaluate which technique is best in terms of patient diagnosis and treatment and how these techniques may fit together to form a complete patient management strategy of diagnosis, stratification, and therapy.

Medical imaging methods and devices are commonly evaluated through computer simulation. Computer-generated phantoms are used to model the patient anatomy and

15 physiology, as well as the imaging process itself [5-7]. Given a model of the imaging process, imaging data of a computer phantom can be simulated as if it were an actual patient. A major advantage to using computer-generated phantoms in simulation studies is that the exact anatomy and physiological functions of the phantom are known, thus providing a gold standard from which to evaluate and improve medical imaging devices and techniques. Realistic computer phantom modeling of human anatomy and function is a vital aspect of simulation.

The 4D NURBS-based cardiac-torso (NCAT) phantom (Figure 1) [5-7] was developed to provide an accurate model of the human anatomy and physiology and is widely used in the 
evaluation of medical imaging devices and techniques. It includes a realistic model for the cardiac and respiratory motions based on tagged MRI data and respiratory-gated CT data, respectively. Both datasets were acquired from normal patients. Combined with accurate models of the imaging process, the 4D NCAT can produce simulated images that mimic those acquired from actual patients. With this ability, the 4D NCAT has gained and continues to gain widespread use in medical imaging research, especially for evaluating and improving cardiac imaging instrumentation, data acquisition techniques and image processing and reconstruction methods [7-9]. It is widely used in myocardial SPECT, providing a tool to study the effects of anatomy and patient motions. The 4D NCAT phantom models the beating heart motion of a particular normal subject using the cardiac motion documented by a set of gated tagged MRI data of a normal male volunteer. One major limitation of the 4D NCAT phantom is that it does not have the ability to realistically simulate abnormal motions due to coronary artery disease. The effects of CAD are simulated to a limited extent by defining ischemic regions resulting from a blockage in the coronary arteries as low perfusion pie-

15 shaped wedges in the NCAT (Figure 1). The motion in this region can be reduced through scaling operations to simulate the altered function of the LV due to the coronary blockage; however, this is a very simple, unrealistic method with no physiological basis.

In the present research, this limitation was overcome by incorporating a physiologically based finite-element (FE) mechanical heart model into the 4D NCAT 20 phantom. Like the original NCAT heart model based on tagged MRI data of a normal subject, the FE model is capable of accurately simulating the normal motion of the left ventricle. The FE model does, however, offer an improved definition of ischemia over the current NCAT implementation in that it provides a more accurate representation of the abnormal motion of 
the LV due to the effects of ischemia. With its physiological basis, the FE model has the flexibility to simulate a wide variety of motion abnormalities of the heart. Defects of any given size and location within the heart can be realistically modeled. Combined with the 4D NCAT, the FE model can be used to produce realistic sets of imaging data from a variety of 5 patients in which the normal or abnormal cardiac function is accurately represented. 


\section{METHODS}

\section{A. Finite Element Model Generation}

A gated high resolution CT image set of a normal male subject acquired on a 32-slice Siemens x-ray multi-slice CT (MS CT) scanner (Siemens AG, Erlangen, Germany) at the

5 Johns Hopkins Hospital was selected and used as the basis for the cardiac geometry of the finite element model. The image data was provided by Dr. Elliot Fishman of the Johns Hopkins Hospital in accordance with the HIPPA standards. The patient study consisted of 9 time frames during the cardiac cycle. Each time frame consisted of a $512 \times 512 \times 231$ image array with a pixel size and slice thickness of $0.421 \mathrm{~mm}$.

The image dataset obtained at the beginning of diastole was used to define the undeformed configuration of the FE model. The boundaries of the epi- and endocardial surfaces of the left ventricle were manually segmented using the software program SURFdriver [10]. The segmented closed contours were used to define a FE mesh of 8,676 nodes for the LV (Figure 2). The FE model for the LV was surrounded by a soft tether mesh

15 represented by an isotropic hypoelastic constitutive model with relatively soft elastic material properties (modulus of elasticity $\mathrm{E}=0.30 \mathrm{KPa}$ and bulk modulus $K=0.12 \mathrm{KPa}$ ) to provide tethering with the outer edges of the image domain. The tether mesh was fully constrained to eliminate rigid body motion. The bulk modulus of the tethering is more than three orders of magnitude less than the bulk modulus of the myocardial wall definition (see below) and modulus of elasticity of the tethering is 25 times less stiff than the modulus of elasticity of the myocardial wall. The tethering, therefore, does not contribute to the strain distribution of the model. This type of constraint has been used previously for this type of cardiac modeling [11]. 
1) Passive Material Model: The LV myocardium was represented as a transversely isotropic hyperelastic material with the fiber angle varying from $-90^{\circ}$ at the epicardial surface, through $0^{\circ}$ at the midwall, to $90^{\circ}$ at the endocardial surface. The strain energy definition for the 5 material describes a material that consists of fibers imbedded in an isotropic ground substance [12]:

$$
W=F_{1}\left(\tilde{I}_{1}\right)+F_{2}(\tilde{\lambda})+\frac{K}{2}[\ln (J)]^{2}
$$

where $F_{1}$ represents the behavior of the matrix surrounding the fibers, $F_{2}$ represents the behavior of the fibers, and the final term represents the bulk (volumetric) behavior, where $K$ is

10 the bulk modulus of the material. $\boldsymbol{F}$ is the deformation gradient tensor. $J$ is the Jacobian and is defined as $J=\operatorname{det}(\boldsymbol{F}) . \quad \tilde{I}_{1}$ is the first deviatoric invariant of the right Cauchy deformation tensor $[13,14]$. The scalar $\tilde{\lambda}$ is the deviatoric stretch ratio along the local fiber direction, $\boldsymbol{a}$.

A neo-Hookean form was used to represent the ground substance matrix:

$$
F_{1}\left(\tilde{I}_{1}\right)=\mu\left(\tilde{I}_{1}-3\right)
$$

15 where $\mu$ is the shear modulus of the ground substance. The passive Cauchy stress tensor is:

$$
\boldsymbol{T}^{(p)}=p \boldsymbol{1}+\frac{2}{J}\left[\left(\tilde{W}_{1} \tilde{\boldsymbol{B}}+\tilde{\lambda} W_{\lambda} \boldsymbol{a} \otimes \boldsymbol{a}\right)-\frac{1}{3}\left(\tilde{W}_{1} \tilde{I}_{1}+\tilde{\lambda} W_{\lambda}\right) \boldsymbol{1}\right]
$$

where $\tilde{W}_{1}$, and $\tilde{W}_{\lambda}$ are strain energy derivatives with respect to $\tilde{I}_{1}$ and $\tilde{\lambda}[14], \tilde{\boldsymbol{B}}$ is the deviatoric left deformation tensor, and “ $\otimes$ ” represents the vector outer product operation.

The stress-stretch behavior for the fiber direction $\left(\tilde{\lambda} W_{\tilde{\lambda}}\right)$ was represented as an exponential, with no resistance to a compressive load: 


$$
\begin{aligned}
& \tilde{\lambda} W_{\lambda}=\tilde{\lambda} \frac{\partial F_{2}}{\partial \lambda}=0, \quad \tilde{\lambda}<1 \\
& \tilde{\lambda} W_{\lambda}=\tilde{\lambda} \frac{\partial F_{2}}{\partial \lambda}=C_{3}\left[\exp \left(C_{4}(\tilde{\lambda}-1)\right)-1\right], \quad \tilde{\lambda} \geq 1 .
\end{aligned}
$$

The material coefficients $C_{3}$ and $C_{4}$ scale the fiber stress and control its rate of rise with increasing stretch, respectively. Details of this constitutive model can be found in Weiss et al. [12].

The material properties of the myocardial wall were determined through least squares fit of biaxial data published by Humphrey [15] which were extrapolated from test data reported by Guccione et al. [16]. The cross-fiber equibiaxial data was used to determine the shear modulus $(\mu)$ of the material model. The equibiaxial test in fiber direction was then used to define the exponential behavior of the material $\left(C_{3}\right.$ and $\left.C_{4}\right) . C_{4}$ was then adjusted until the

10 radial displacement of the epicardium was approximately $3 \mathrm{~mm}$. This $3 \mathrm{~mm}$ radial displacement was the average of the radial displacement documented in the gated CT image data from which the geometry was defined. The material coefficients for the passive LV were $\mu=2.50 \mathrm{kPa}, C_{3}=0.27 \mathrm{kPa}$, and $C_{4}=21.0$. A bulk modulus $K$ of $195.00 \mathrm{kPa}$ was used in all of the FE models and represented the highest bulk modulus that did not cause numerical 15 instability in the model due to volumetric locking [13]. Using this value for the bulk modulus, the average change in relative volume was less than $6 \%$ for all of the models in this study [17].

2) Elastance Active Contraction Model: A time varying "elastance" active contraction model $[18,19]$ was used to yield a constitutive model that could simulate both the passive mechanics 20 of diastole and the active contraction during systole. The total Cauchy stress $T$ is defined as the sum of the active stress tensor $T^{(a)}(\boldsymbol{a} \otimes \boldsymbol{a})$ and the passive stress tensor $T^{(p)}$ : 


$$
T=T^{(p)}+T^{(a)}
$$

where $\boldsymbol{a}$ is the deformed fiber vector (unit length), defined as $\lambda \boldsymbol{a}=\boldsymbol{F} \cdot \boldsymbol{a}_{0}$. The time varying elastance model is a modification of the standard Hill equation that scales the standard equation by the variable $C_{t}$ which governs the shape of the activation curve [19]. The active $5 \quad$ fiber stress $T^{(a)}$ is defined as

$$
T^{(a)}=T_{\max } \frac{C a_{0}^{2}}{C a_{0}^{2}+E C a_{50}^{2}} C_{t},
$$

where $T_{\max }=135.7 \mathrm{KPa}$ is the isometric tension under maximal activation at the peak intracellular calcium concentration of $C a_{0}=4.35 \mu \mathrm{M}$. The length dependent calcium sensitivity is governed by the following equation:

$$
E C a_{50}=\frac{\left(C a_{0}\right)_{\max }}{\sqrt{\exp \left[B\left(l-l_{0}\right)\right]-1}},
$$

where $\left(C a_{0}\right)_{\max }=4.35 \mu \mathrm{M}$ is the maximum peak intracellular calcium concentration, $B=4.75$ $\mu \mathrm{m}^{-1}$ governs the shape of the peak isometric tension-sarcomere length relation, $l_{0}=1.58 \mu \mathrm{m}$ is the sarcomere length at which no active tension develops, and $l$ is the sarcomere length which is the product of the fiber stretch $\lambda$ (deformed length/reference length) and the

15 sarcomere unloaded length $l_{r}=2.04 \mu \mathrm{m}$. A detailed description of this model can be found in Guccione et al. [18,19].

In the FE implementation, the active contraction is governed by the product $T_{\max } C_{t}(\mathrm{an}$ active contraction stress) in (6), which was used to define a "load curve", specifying the degree of contraction and subsequent relaxation during the cardiac cycle (Figure 3A). The intraventricular systolic pressure-time curve from Guccione et al. [19] was used with the diastolic portion of the pressure-time curve corresponding to passive filling [20] (Figure 3B). 
The analyses were conducted using NIKE3D, a non-linear, large deformation FE package [21]. Transmural strain distributions were determined for the entire normal LV. The predicted FE deformations were exported to the NCAT model in order to create the synthetic myocardial SPECT image data sets

5 3) Modeling Regional Ischemia: Ischemic regions in the FE model are simulated as detailed by Mazhari et al. [22]. The length dependent calcium sensitivity (7) was reduced by increasing the intracellular calcium concentration $\left(\mathrm{Ca}_{0}\right)_{\max }$ from $4.35 \mu \mathrm{M}$ to $7.90 \mu \mathrm{M}$ in the ischemic regions. This change in calcium concentration, which causes a complete cessation of the contractile function within the ischemic region, can be made by changing a single variable

10 in the material model definition within the finite element model input file. The passive diastolic function of the myocardium remained unchanged, as this is dependent entirely upon the passive constitutive model definition. The ischemic regions were defined to be similar in size and shape as depicted in Figure 1B and were exactly the same size and shape in all of the analyses. The computations were carried out on a Compaq DS20E SMP computer, consisting

15 of two $667 \mathrm{MHz}$ Alpha $21264 \mathrm{CPUs}$ and 4 GB of core memory requiring approximately an hour and a half of analysis time. This relatively short analysis time allowed for multiple iterations of the analyses for the normal model. For example, this type of iterative analysis was used to reproduce epicardial radial deformation through modification of the $C_{4}$ parameter of the material model. used to create the geometry and mesh (TrueGrid, XYZ Scientific Applications, Livermore, CA). Relatively simply modifications to the size of the ischemic region can be made with ease, with less than 5 minutes in development time. Changing the shape of the ischemic 
region requires more development time (on the order of several hours) to modify the FE discretization to conform to the new geometry.

\section{B. Evaluation of the Finite Element Model}

Three cases were modeled using the FE representation of the LV described above, a normal model to serve as a baseline and two pathological models to study the effects of ischemia on cardiac function. In order to determine the accuracy of the normal FE model, the gross deformations (wall thickening, twist, etc.) of the model were compared with the gross deformations of the NCAT phantom, which are based on tagged MRI data of a normal male subject. In the first pathological model, a subendocardial anterior ischemic region was defined. A second ischemic model was created with a transmural ischemic region defined in the same location as the subendocardial ischemia model. The subendocardial ischemic region extended through half the thickness of the myocardium (Figure 1), while the transmural ischemic region encompassed the full thickness of the wall. The strain distributions for a

15 single cross-section through the ischemic regions were compared for the three FE models. The mean and standard deviations for the fiber, radial and circumferential strains within the ischemic regions were determined for each case. The transmural strain distributions were compared within ischemic regions.

The deformation results based on the FE model predictions for myocardial ischemia

20 were compared with deformation results obtained using the simple definition of ischemia within the NCAT simulation. In the current version of the NCAT phantom, ischemic regions are modeled by just limiting or eliminating the motion of the LV myocardium in the region. 
The transmural ischemia region FE mechanical model was adjusted to model this simple definition of ischemia for two cases. In the first case, the nodes in the ischemic region were set to move $50 \%$ as much as they would in the normal FE mechanical model over the entire cardiac cycle. In the second case, the displacements within the ischemic region were 5 completely eliminated $(100 \%$ reduction) over the entire cardiac cycle. These two cases represent the most common traditional definitions of ischemia used in 4D NCAT simulations [23]. The active contraction within the ischemic region of the FE model as well as the pressure load on the endocardial surface were eliminated so that the displacement based boundary conditions were the only loads on this region. The strain distributions were then compared with the strain distributions determined for the transmural ischemia FE model described above. Specifically, the radial, circumferential, in-plane shear (shear strains within the short-axis plane) and the fiber strain were compared. The fiber strains are defined as the strain along the direction of the defined fibers in the model.

The organ models in the NCAT phantom are defined using 3D cubic NURBS surfaces $[5,24,25]$. The size and shape of each NURBS surface model is determined by a set of control points with each control point having a weight of 1 . A surface can be altered by applying affine and other transformations to the control points in order to model anatomical variation or patient motion. The motion of the NCAT heart was modeled by defining time-position cubic NURBS curves for each of the control points of the heart surfaces creating timedependent 4D NURBS surfaces. The time curves were originally derived from a gated tagged 
MRI dataset of a normal human volunteer. The 3D positions of the control points over the cardiac cycle were determined from the motion of the taglines in the dataset. A cubic NURBS curve was then fit to the time-changing location for each control point using established methods for interpolating a 3D NURBS curve to a given set of points in space [24,25]. From

5 the continuous time-position curves defined for the control points, an infinite number of time frames over the cardiac cycle can be produced. Time samples in between the pre-defined locations of the control points obtained from the tagged MRI analysis are simply obtained through cubic spline interpolation.

The FE mechanical model of the LV was converted to 4D NURBS surfaces, which were then incorporated into the NCAT cardiac model. The LV surface of the NCAT phantom was setup so that the control points which define it lie approximately on the surface (within $0.02 \mathrm{~mm}$ 's to their corresponding surface points). Given this approximate one-to-one correspondence, the node points of the FE mesh at time 0 were used to define the control points for an inner, outer, and five mid-ventricular NURBS surfaces for the LV (Figure 4).

15 The motion of the node points over the cardiac cycle as determined by the FE analysis were used to define the time-position cubic NURBS curves for the control points creating timevarying $4 \mathrm{D}$ NURBS surfaces for the LV. These surfaces replaced the previous LV beating heart model of the NCAT phantom. The motion of the 4D NURBS surfaces originally defined for the atria were modified to accommodate the new ventricular surfaces created from the 20 mechanical model. For each subsequent time frame, the atria models are manipulated to fit the motion of the ventricles and to fit a volume curve for a normal, healthy human male [26]. The atria are first translated downward by an amount equal to the longitudinal contraction of the ventricles. The atria are then scaled longitudinally $(\mathrm{z})$ depending on the amount of 
longitudinal contraction by the ventricles. The origin for this scaling operation is set to be the bottom center of each atria model. The height of the atria is increased by this scaling operation by an amount equal to the amount of longitudinal contraction of the ventricles. Once the atria are scaled longitudinally, the control points located at the bottom of each atria model are then set to the control points of the corresponding ventricle model to blend the two models together. The atria models are then scaled transversely in the x- and y-directions until they fit a certain volume on the predetermined volume curve from a normal male.

A software application was developed to perform the above tasks seamlessly. Currently, the beating heart is defined in the NCAT software by a time series of heart files. Each file contains the 3D NURBS surfaces defined for the cardiac structures at a specific time point in the cardiac cycle. The phantom application reads in the time files describing the heart's motion over one cardiac cycle, then fits cubic 3D NURBS curves to the time changing position of each structure's control points using the spline interpolation methods of Piegl et al. $[24,25]$. The program then generates the beating heart at any time point in its cycle

15 (Figure 5). A software application was developed that reads in the 4D NCAT heart files and the output of the FE analysis consisting of the ventricular node points and their movement in time, converts this information into new 4D NURBS surfaces for the ventricles and atria, and saves these surfaces into a new time series of heart files that can be read by the NCAT phantom application. By establishing this link, the FE computational heart model can be used

20 to produce numerous datasets representing healthy and diseased hearts for use in medical imaging research.

\section{Myocardial SPECT Simulation Study Using the FE-NCAT}

Using the technique described above, the FE mechanical models for the normal heart 
and ischemic hearts were incorporated into the 4D NCAT phantom. To demonstrate the use of the improved phantom, a myocardial SPECT simulation was performed to investigate how the differences in contractile function between the subendocardial and transmural infarcts manifest themselves in myocardial SPECT images. Using the 4D NCAT, 16 time frame

5 phantoms were generated each modeling the radioactivity concentrations and attenuation distributions in the different organs over the cardiac cycle ( 1 beat per second). The distribution of radioactivity concentration was set to model the uptake of a typical Tc-99m sestamibi patient study [27].

Each generated $3 \mathrm{D}$ phantom was stored in a $128 \times 128 \times 128$ array with a pixel size and

10 slice thickness of $0.31 \mathrm{~cm}$. For each heart model, the 16 time frames were averaged to create a phantom of the average beating heart motion for that case. For each phantom, emission projection data were generated using a realistic parallel projection model simulating an Lconfigured dual-camera SPECT system equipped with a transmission source. A complete projection dataset was generated over the typical $180^{\circ}\left(45^{\circ}\right.$ right anterior oblique to $45^{\circ}$ left

15 posterior oblique) rotational arc around the patient. The simulations used a projection model that included the effects of non-uniform attenuation, detector response, and scatter. A lowenergy high-resolution (LEHR) collimator with a thickness of $4.1 \mathrm{~mm}$ and hexagonal holes with a flat-to-flat size of $0.19 \mathrm{~mm}$ was simulated. The $128 \times 128$ simulated emission projection images were collapsed to $64 \times 64$ to simulate sampling used in a clinical data acquisition.

The emission projection data were reconstructed using the iterative ordered subset expectation maximization (OS-EM) reconstruction method with compensation for attenuation, scatter, and collimator-detector response [28]. The attenuation phantoms 
generated to simulate each case were used to compensate for the effects. The images were reconstructed into $64 \times 64$ arrays with 64 slices and a pixel width and slice thickness of 0.63 $\mathrm{cm}$. The ejection fractions, wall thickening, long-axis contraction were calculated within the NCAT phantom for the all of the simulations based on the FE models.

The SPECT images based on the FE models simulating subendocardial and transmural ischemia were simulated in the $4 \mathrm{D}$ NCAT as having a $20 \%$ and $50 \%$ reductions in perfusion in the ischemic regions respectively. This allowed for evaluation of both the abnormal motion produced by the FE models as well as the effects of changes in perfusion in the simulated images. These images were compared with the normal FE based NCAT SPECT 10 simulation.

The FE based ischemia simulations were further compared with simulated SPECT images based on the simple definition of ischemia available in the NCAT phantom. These image data sets were based on the ischemia cases in which the wall motion is reduced by $50 \%$ as well as the fixed case in which there is $100 \%$ reduction in wall motion. All of the 15 simulated SPECT images were defined as having normal perfusion. Image intensity profiles were taken across the anterior (ischemic) regions thus providing a means to compare changes in intensity due to alterations in geometry alone. 


\section{RESULTS}

\section{A. Analysis of the Normal Finite Element Model}

The motion of the normal FE LV mechanical model compared favorably to that

5 previously defined in the NCAT phantom which is based on tagged MRI data from a normal male subject. The 4D NCAT heart model accurately represents the contracting motion of the heart that has been previously described using tagged MRI analysis [29-33]. As it contracts, the beating heart exhibits a wringing-like twisting motion of the left ventricle and radial (wall thickening) and longitudinal (base to apex) contraction of the heart walls. The twisting motion of the LV consists of the clockwise rotation of the base and a counterclockwise rotation of the apex during systole (Figure 6). The LV twisting motion and radial contraction of the normal FE model were found to be very similar to that of the 4D NCAT cardiac model. The longitudinal contraction of the FE model was slightly smaller than that found in the 4D NCAT cardiac model (Table 1). However, it was identical to that observed in the gated

15 MSCT data upon which it was based.

The normal FE model had an ejection fraction of $62 \%$, which is in the range for healthy patients $(55-75 \%)$ [34]. The highest radial and circumferential transmural strains occurred at the endocardium with a near linear decrease from endocardium to epicardium (Figure 7). In contrast, the fiber stretch maximum occurred at the mid-wall. The longitudinal

20 strains and in plane shear strains showed a flat distribution throughout the wall with average strains of 0.10 and 0.01 , respectively. 


\section{B. Analysis of the Ischemic Finite Element Models}

The two ischemic (subendocardial and transmural) LV FE mechanical models showed a reduced wall thickening in the anterior region of the LV (Table 1), the location of the defect. The FE LV model with the transmural defect had the largest reduction in wall thickening. As a result, the motion abnormality of the transmural defect was more pronounced when viewing animations of the models. The defects did not affect the longitudinal contraction of the LV base due to their mid-ventricular location.

Both the subendocardial ischemia and the transmural ischemia FE models demonstrated reduced ejection fractions (58\% and 55\%, respectively). The average

10 transmural fiber strain in the subendocardial ischemic region changed from a contractile value of -0.09 to a tensile value of 0.03 (Figures 8 and $9 \mathrm{~A}$ ). The transmural ischemic region produced an average tensile fiber strain of 0.05 with the mid-wall having a near constant fiber strain of 0.11 (Figure 9A). The average circumferential strain was -0.12 in the normal model, 0.02 for the subendocardial ischemia model and 0.13 in the transmural ischemic model

15 (Figures 8 and 9B). Similarly, the average radial strain was 0.18 in the normal model, 0.09 in the subendocardial ischemia model and 0.01 in the transmural ischemia model (Figures 8 and 9C).

\section{Comparison of the FE Ischemic Models to Those Derived from the Current 4D NCAT} Phantom

The comparison of the displacements of the subendocardial and transmural FE models with the displacements determined from the simple definition of ischemia in the 4D NCAT simulation show that the NCAT ischemic region had strain distributions that were relatively 
low in magnitude throughout these regions (Figures 10 and 11). The 50\% reduction in displacement indicated that the fiber strain resulting from this ischemia definition would show less than $8 \%$ average strain transmurally with other strain measures showing less than $5 \%$ strain. The fixed case $(100 \%$ reduction in displacement) would produce strains that were

5 essentially zero in magnitude for all measures of strain (Figures 10 and 11).

\section{Myocardial SPECT Simulation Study}

The subendocardial ischemia FE based NCAT simulations showed little discernable difference with respect to the normal model regardless of the level of perfusion $(20 \%$ or $50 \%$

10 reduction) used in the simulation (Figure 12). The transmural simulations demonstrate that the wall thinning in the ischemic anterior region is apparent even in the case where the perfusion has been reduced by only $20 \%$. These reconstructed short-axis images are shown for each heart model at the end-systolic time frame.

The FE-based simulations were found to produce differences that were observable

15 even using low-resolution myocardial SPECT. Figure 13 illustrates how the differences in the two ischemia definitions (the simple definition implemented in the 4D NCAT and the physiologically-based definition in the FE models) manifest themselves visually in simulated myocardial SPECT images. The FE based NCAT simulation shows an appreciable reduction in the vertical intensity profile across the wall while the profiles of the other models show 20 relatively little (Fig. 13 top) reduction in intensity. The difference in vertical intensity is due to the wall thinning evident in the transmural FE model (Fig. 13 bottom). 


\section{DISCUSSION}

We developed a functionally realistic FE mechanical model of the LV to simulate normal and abnormal functions of the beating heart with the purpose of incorporating it into

5 the 4D NCAT phantom for use in cardiac imaging research. Three different cases, one normal and two pathological (subendocardial and transmural anterior ischemic regions) were simulated and analyzed to evaluate the FE model. The normal FE model produced an ejection fraction within the range for a normal patient (62\%) while the ischemic models showed reduced ejection fractions, (58\%) and (55\%) for the subendocardial and transmural defects respectively (Table 1).

The radial, circumferential and longitudinal strain distributions of the three models showed similar trends as reported in the literature. Fiber strain predictions from the normal FE model were in good agreement with the predictions of the cylindrical numerical model developed by Guccione et al. [19]. The maximum fiber contraction for the normal model was

$15-0.09$ (referenced to the beginning of diastole) and showed little variation transmurally while the Guccione model showed a maximum of -0.06 using the same reference configuration. The maximum fiber strain value for the Guccione model was at the midwall with the fiber strain values decreasing toward the epi- and endocardial surfaces. The predicted transmural fiber strain distributions for the normal FE model and fiber strain distribution within the ischemic

20 region of the transmural FE model were non-uniform. Uniform fiber strain distributions have been reported in both the normal $\operatorname{LV}[35,36]$ and in stunned canine myocardium strain 
myocardium [37]. This discrepancy is likely due to the assumed non-patient specific fiber distributions used in the models [38-40].

The normal FE model predicted a maximum average systolic radial strain of 0.33 at the endocardium (referenced to end-diastole) decreasing to a value of 0.15 at the epicardium

5 (Figure 6), The Guccione model showed a similar radial strain distribution with the maximum average radial strain value of 0.40 also occurring at the endocardium and decreasing to a value of 0.26 at the epicardial surface again referenced to end-diastole. The circumferential strain distributions were also similar between the two models. The FE model had a maximum average circumferential systolic strain of -0.30 at the endocardium which decreased to a value of -0.08 at the epicardium. The Guccione model had a maximum circumferential strain of 0.20 at the endocardium decreasing to a value of -0.14 at the epicardium. The differences in strain values are likely due to the use of a realistic geometry in the present study as opposed to the cylindrical geometry used in the referenced study.

The average LV fiber strain (-0.16 \pm 0.08$)$, referenced to end-diastole, was higher than

15 that reported by Tseng et al. $(-0.12 \pm 0.01)$ [35] for the mid-ventricle but consistent to the values reported by MacGowan et al. (-0.15) [36] for the entire LV. The change in circumferential, in-plane shear and radial strain predictions were also consistent with measured values found in the literature (Table 2). The six unique measures of strain determined from the normal FE model were found to be comparable with strain data (Table 3) from the canine study of Waldman et al. [41]. However, the predicted circumferential strain at the endocardium was found to be higher than reported in the referenced study.

The transmural fiber strains predicted by the ischemic FE model were consistent with those reported for an ischemic (stunned) dog model [37] (Figure 8). Mazhari et al. measured 
transmural fiber systolic strains of approximately $14.8 \%$. These values compare well with the $12 \%$ transmural strains found in the mid-wall of the transmural ischemia model (Figure $8 \mathrm{~A}$ ). However, the average value for the transmural ischemic region was 0.05 due to the decreases in fiber strain at the endo- and epicardial walls. This non-uniformity of the strain distribution 5 is likely due to the assumed fiber distributions used in the models [38-40]

The transmural circumferential strain results were consistent with the results of the canine ischemia study results of Kraitchman et al. [42] compared with the normal contractile value of $15 \%$ (Figure $8 \mathrm{~B}$ ). A canine ischemia study by looked at using fast harmonic phase (FastHARP) MRI to detect the onset of ischemia. For this study, they measured ischemia as a $50 \%$ reduction in myocardial blood flow as determined by microsphere measurements. Their strain measurement results indicated that ischemic tissue showed tensile circumferential strain values of $10-18 \%$ immediately following the onset of ischemia ( $<40 \mathrm{sec}$ from onset) in contrast to the contractile value of $18 \%$ measured in the normal tissue distant from the ischemic region. Their results compare well with our transmural ischemia model which 15 predicted an average tensile value of $16 \%$ strain in the ischemic region.

The use of material coefficients obtained by fitting mechanical data of normal myocardium in order to model stunned myocardium appears to be justified. Recent work by Pislaru et al. [43] indicated that there is no difference in the passive material behavior of normal and stunned myocardium. Using strain echocardiography, they estimated the tissue 20 stiffness by determining the relationship between the intraventricular pressure and the change in wall thickness and found that the stiffnesses of stunned myocardium and normal remote myocardium were not significantly different. In contrast, infarcted myocardium was 
substantially stiffer than stunned or normal myocardium, with infarcted tissue showing little deformation during diastolic loading.

The systolic contractile behavior of stunned myocardium appears to be indistinguishable from necrotic myocardium using the imaging modalities of cine MRI and tagged MRI. Juergens et al. [44] looked at the changes in global measures such as ejection fraction as well as local measures such as changes in principal strains in normal, ischemic and infarcted myocardium during systole as measured by tagged MRI strain analysis. Both stunned and infarcted (necrotic) tissue were easily distinguishable from normal tissue due to the lack of contraction in these tissue types. However, the behavior of nectrotic and stunned tissue as measured by changes in the maximum and minimum in-plane (short axis) principal strains were not statistically different from one another. Thus, the studies of Pislaru [43] and Juergens [44] provide the framework for the development of a FE based 4D NCAT simulation for infarction. The passive properties of infarcted tissue could be estimated by reproducing the wall stiffening measured by Pislaru in the FE model, while the systolic behavior of 15 infarcted myocardium could be modeled as ischemic, as done in the present study.

The normal and abnormal FE models showed reasonable agreement with strain measurements reported in the literature. However, several limitations of the FE model should be acknowledged. Recent work suggests that orthotropic material models provide a more accurate representation of the passive material behavior of the myocardium than a transverse

20 isotropic model such as was used in the current study. Usyk et al. [48] found that the use of an orthotropic material model in a finite element model provided better agreement with experimental end-diastolic strain distributions than a transversely isotropic material model. The authors stated that they believed that the contribution of the orthotropic passive material 
definition to end-systolic strains was likely small, however, they found that the addition of an active stress transverse to the muscle fibers greatly improved the agreement between measured and modeled transverse end-systolic shear strains. Biaxial tests on myocardial tissue by Lin et al. [49] suggested that this in-plane, cross-fiber contractile stress was approximately $40 \%$ of the muscle fiber stress and was likely to contribute to the end-systolic strain distributions. Recent work by Walker et al. [50] indicated that cross-fiber active contraction stress was necessary for the accurate prediction of end-systolic strain distributions. In this study, the strain measurements from tagged MRI analysis of sheep LV with MI induced ventricular aneurysms were compared with subject-specific FE LV models. The inclusion of in-plane, cross-fiber active contraction stress reduced the overall RMS error in strain from $7.4 \%$ to $5.4 \%$, a $27 \%$ decrease in error. In the future, cross-fiber active contraction stress components will be added to the active contraction material model presented above in order to improve our strain predictions.

Studies in the literature have reported uniform fiber stretch distributions [35,45-47]

15 while the FE model predicted mid-wall fiber stretches were higher than those at the endo- and epicardial surfaces (Figures 5 and 8). It should also be noted that while the FE model geometry was patient-specific, the fiber angle distribution was idealized. It is likely that the assumed fiber angle distribution directly contributed to the non-uniformity of the transmural strain distribution in the normal LV results as well as the transmural ischemic results. Work is 20 currently underway to allow for the use of realistic fiber distribution definitions based on DTMRI and the incorporation of an orthotropic material definition into the FE models.

Despite these limitations, the motion of the normal FE model was found to be very similar to that defined in the normal NCAT heart model, which illustrates the same 
contracting, wringing motion of the heart observed in tagged MRI studies of normal patients. The two ischemic FE models showed no overall contraction in the location of the defect, with the transmural defect showing circumferential elongation and wall thinning. As mentioned above, the abnormal FE mechanical models were found to produce a more accurate

5 simulation for the cardiac motion abnormalities as compared to the original NCAT phantom. The NCAT definition may be more appropriate for modeling infarcted regions in which the scar tissue is relatively stiff compared with the normal tissue and thus would undergo little passive deformation [43] or in the $50 \%$ displacement case where the ischemic tissue retains some contractility.

These studies indicate that a continuum based mechanical model is necessary to reproduce realistic deformations within the ischemic regions. The non-FE based ischemia definition does not produce the circumferential elongation and wall thinning found ischemic regions. Nor does simply reducing the contraction in the ischemic regions reproduce the subtleties of the subendocardial ischemia where the normally contracting epicardium and

15 subepicardium contracts against the passive ischemic subendocardium. These geometric changes also cause a reduction in the intensity levels above that resulting from the ischemia induced reduction in perfusion (Fig. 13), as illustrated by the intensity profile across the wall from the model simulating the transmural defect. Despite its shortcomings, the NCAT definition ischemia is likely to be more appropriate for modeling infarcted regions in which 20 the scar tissue is relatively stiff compared with the normal tissue and thus would undergo little passive deformation [43] or in the $50 \%$ displacement case where the ischemic tissue retains some contractility. 
The normal and abnormal FE mechanical models were incorporated into the NCAT phantom and used in a simulation study to investigate how the contractile differences between the subendocardial and transmural infarcts manifest themselves in myocardial SPECT images. Ex vivo studies have documented that the blood flow and metabolism requirements for the 5 subendocardium can be markedly different than for the sub-epicardium [51-56]. The vulnerability to ischemia for the two regions is likewise dissimilar. Cell death due to ischemia is thought to begin in the subendocardial layer and then develop as a wavefront towards the endocardium [57,58]. Therefore, it is of great clinical importance to be able to detect differences in in vivo blood flow, metabolism and other properties between the subendocardium and sub-epicardium. There is evidence that the transmural extent of ischemic damage in the myocardium due to subendocardial ischemia $[59,60]$ as shown by blood flow can be a predictor for return of contractile function following coronary artery reperfusion [61]. Currently, only MRI techniques are beginning to emerge that would allow transmural functional characterization of some aspects of the myocardium $[59,60]$. However, it is

15 unlikely that MR techniques will adequately address such issues as differentiated lipid and glucose metabolism and ligand binding. This is where nuclear medicine techniques such as SPECT can be very useful. As demonstrated with the NCAT simulations, at the resolutions currently in use, SPECT would have difficulty in the delineation of subendocardial ischemia from normal myocardium. There was relatively little visible difference between the normal 20 myocardium SPECT image and the subendocardial ischemic SPECT images (Figures 12, 13). The simulation results demonstrate the great potential that the FE-NCAT combination has for use in cardiac imaging research. 
Incorporation of the FE mechanical model into the 4D NCAT phantom provides a more realistic, physiological basis for the cardiac motion of the phantom. The FE mechanical cardiac model combined with the 4D NCAT phantom and other simulation tools, developed in this work is capable of providing realistic, predictive imaging data of a patient population 5 with varying whole-body anatomy and healthy and diseased states of the heart. With its physiological basis, the FE model has the flexibility to simulate a wide variety of motion abnormalities of the heart. With the ability to simulate imaging data consistent to that from actual patients, the enhanced 4D NCAT phantom will provide a vital simulation tool in medical imaging research, supplying a known truth from which to evaluate and improve existing and emerging cardiac imaging methods that assess cardiac function through measurements of myocardial deformation. 


\section{ACKNOWLEDGEMENTS}

The authors thank Dr. Elliot Fishman, Department of Radiology, The Johns Hopkins University, for providing the images used to define the LV geometry. This work was supported in part by the National Institutes of Health under Grants R01 EB00121 and R01

5 HL71253, by the National Science Foundation under grant BES-0134503, and by the Director, Office of Science, Office of Biological and Environmental Research, Medical Sciences Division of the U.S. Department of Energy under contract DE-AC03-76SF00098. 


\section{REFERENCES}

[1] K. Morris, S. Palmeri, and R. Califf, "Value of radionuclide angiography for predicting cardiac events after acute myocardial infarction," American Journal of Cardiology, vol. 55, pp. 318-324, 1985.

5 [2] L. Shaw, S. Heinle, S. Borges-Neto, K. Kesler, R. Coleman, and R. Jones, "Prognosis by measurements of left ventricular function during exercise," Journal of Nuclear Medicine, vol. 39, pp. 140-146, 1998.

[3] H. White, R. Norris, M. Brown, P. Brandt, R. Whitlock, and C. Wild, "Left ventricular end-systolic volume as the major determinant of survival after recovery from myocardial infarction," Circulation, vol. 76, pp. 44-51, 1987.

[4] S. Johnson, C. Bigelow, K. Lee, D. Pryor, and R. Jones, "Prediction of death and myocardial infarction by radionuclide angiocardiography in patients with suspected coronary artery disease," American Journal of Cardiology, vol. 67, pp. 919-926, 1991.

[5] W. P. Segars, "Development of a new dynamic NURBS-based cardiac-torso (NCAT) phantom, PhD dissertation," The University of North Carolina, May 2001.

[6] W. P. Segars, D. S. Lalush, and B. M. W. Tsui, "A realistic spline-based dynamic heart phantom," IEEE Transactions on Nuclear Science, vol. 46, pp. 503-506, 1999.

[7] W. P. Segars, D. S. Lalush, and B. W. M. Tsui, "Modeling respiratory mechanics in the MCAT and spline-based MCAT phantoms," IEEE Transactions on Nuclear Science, vol. 48, pp. 89-97, 2001. 
[8] W. P. Segars and B. M. W. Tsui, "Study of the efficacy of respiratory gating in myocardial SPECT using the new 4-D NCAT phantom," IEEE Transactions on Nuclear Science, vol. 49, pp. 675-679, 2002.

[9] B. M. W. Tsui, W. P. Segars, and D. S. Lalush, "Effects of upward creep and respiratory motion in myocardial SPECT," IEEE Transactions on Nuclear Science, vol. 47, pp. 1192-1195, 2000.

[10] www.surfdriver.com.

[11] A. I. Veress, G. T. Gullberg, and J. A. Weiss, "Measurement of Strain in the Left Ventricle during Diastole with cine-MRI and Deformable Image Registration," Journal of Biomechanical Engineering, vol. 127, pp. 1195-1207, 2005.

[12] J. A. Weiss, B. N. Maker, and S. Govindjee, "Finite element implementation of incompressible, transversely isotropic hyperelasticity," Computer Methods in Applied Mechanics and Engineering, vol. 135, pp. 107-128, 1996.

[13] K.-J. Bathe, Finite Element Procedures in Engineering Analysis. Englewood Cliffs: Prentice-Hall, 1982.

[14] A. Spencer, Continuum Mechanics. New York: Longman, 1980.

[15] J. D. Humphrey, Cardiovascular solid mechanics cells, tissues and organs. New York: Springer-Verlag, 2002.

[16] J. M. Guccione, A. D. McCulloch, and L. K. Waldman, "Passive material properties of intact ventricular myocardium determined from a cylindrical model," Journal of Biomechanical Engineering, vol. 113, pp. 42-55, 1991. 
[17] F. C. Yin, C. C. Chan, and R. M. Judd, "Compressibility of perfused passive myocardium," American Journal of Physiology-Heart and Circulation Physiology, vol. 40, pp. H1864-H1870, 1996.

[18] J. M. Guccione and A. D. McCulloch, "Mechanics of active contraction in cardiac muscle: Part I-constitutive relations for fiber stress that describe deactivation," Journal of Biomechanical Engineering, vol. 115, pp. 72-81, 1993.

[19] J. M. Guccione and A. D. McCulloch, "Mechanics of active contraction in cardiac muscle: Part II-constitutive relations for fiber stress that describe deactivation," Journal of Biomechanical Engineering, vol. 115, pp. 82-90, 1993.

[20] R. M. Berne and M. N. Levy, Physiology. St. Louis, MO: Mosby Year Book, 1998.

[21] B. N. Maker, R. M. Ferencz, and J. O. Hallquist, "NIKE3D: A nonlinear, implicit, three-dimensional finite element code for solid and structural mechanics," Lawrence Livermore National Laboratory Technical Report, vol. UCRL-MA, \#105268, 1990.

[22] R. Mazhari, J. H. Omens, J. W. Covell, and A. D. McCulloch, "Structural basis of regional dysfunction in acutely ischemic myocardium," Cardiovascular Research, vol. 47, pp. 284-93, 2000.

[23] W. P. Segars, B. M. Tsui, D. S. Lalush, E. C. Frey, M. A. King, and D. Manocha, "Development and application of the new dynamic Nurbs-based Cardiac-Torso (NCAT) phantom," Journal of Nuclear Medicine, vol. 42, pp. 7P-7P, 2001.

20 [24] L. Piegl, "On NURBS: a survey.," IEEE Computer Graphics and Applications, vol. 11, pp. 55-71, 1991.

[25] L. Piegl and W. Tiller, The Nurbs Book. New York: Springer-Verlag, 1997.

[26] M. Guyton, Textbook of Medical Physiology. Philadephia: W.B. Saunders Co., 2000. 
[27] W. D. Leslie, S. A. Tully, M. S. Yogendran, L. M. Ward, K. A. Nour, and C. J. Metge, "Prognostic value of automated quantification of $99 \mathrm{mTc}$-sestamibi myocardial perfusion imaging," J Nucl Med, vol. 46, pp. 204-11, 2005.

[28] H. M. Hudson and R. S. Larkin, "Accelerated image reconstruction using ordered subsets of projection data," IEEE Transactions on Medical Imaging, vol. 13, pp. 601609, 1994.

[29] J. Park, D. N. Metaxas, and L. Axel, "Analysis of left ventricular wall motion based on volumetric deformable models and MRI-SPAMM," Medical Imaging Analysis, vol. 1, pp. 53-71, 1996.

[30] J. Park, D. N. Metaxas, and L. Axel, "Quantification and visualization of the 3D nonrigid motion of the left ventricle," in Proceedings of the SPIE Medical Imaging Conference (Physiology and Function), pp. 177, 1997.

[31] J. Park, D. N. Metaxas, A. A. Young, and L. Axel, "Deformable models with parameter functions for cardiac motion analysis from tagged MRI data," IEEE Transactions on Medical Imaging, vol. 15, pp. 278-289, 1996.

[32] A. A. Young and L. Axel, "Three-dimensional motion and deformation of the heart wall: estimation with spatial modulation of magnetization--a model-based approach," Radiology, vol. 185, pp. 241-7, 1992.

[33] A. A. Young, D. L. Kraitchman, L. Dougherty, and L. Axel, "Tracking and finite element analysis of stripe deformation in magnetic resonance tagging," IEEE Transactions on Medical Imaging, vol. 14, pp. 413-421, 1995.

[34] D. L. Kasper, Braunwald E., A. Fauci, S. Hauser, D. Longo, and J. J. L., Harrison's Principles of Internal Medicine, vol. 16: McGraw-Hill Professional, 2004. 
[35] W. Y. Tseng, T. G. Reese, R. M. Weisskoff, T. J. Brady, and V. J. Wedeen, "Myocardial fiber shortening in humans: initial results of MR imaging," Radiology, vol. 216, pp. 128-39, 2000.

[36] G. A. MacGowan, E. P. Shapiro, H. Azhari, C. O. Siu, P. S. Hees, G. M. Hutchins, J. L. Weiss, and F. E. Rademakers, "Noninvasive measurement of shortening in the fiber and cross-fiber directions in the normal human left ventricle and in idiopathic dilated cardiomyopathy," Circulation, vol. 96, pp. 535-41, 1997.

[37] R. Mazhari, J. H. Omens, R. S. Pavelec, J. W. Covell, and A. D. McCulloch, "Transmural distribution of three-dimensional systolic strains in stunned myocardium," Circulation, vol. 104, pp. 336-41, 2001.

[38] T. Arts, K. D. Costa, J. W. Covell, and A. D. McCulloch, "Relating myocardial laminar architecture to shear strain and muscle fiber orientation," American Journal of Physiology - Heart and Circulatory Physiology, vol. 280, pp. H2222-9, 2001.

[39] J. Rijcken, P. H. Bovendeerd, A. J. Schoofs, D. H. van Campen, and T. Arts, "Optimization of cardiac fiber orientation for homogeneous fiber strain during ejection," Annals of Biomedical Engineering, vol. 27, pp. 289-97, 1999.

[40] J. Rijcken, P. H. Bovendeerd, A. J. Schoofs, D. H. van Campen, and T. Arts, "Optimization of cardiac fiber orientation for homogeneous fiber strain at beginning of ejection," Journal of Biomechics, vol. 30, pp. 1041-9, 1997.

[41] L. K. Waldman, D. Nosan, F. Villarreal, and J. W. Covell, "Relation between transmural deformation and local myofiber direction in canine left ventricle," Circulation Research, vol. 63, pp. 550-62., 1988. 
[42] D. L. Kraitchman, S. Sampath, E. Castillo, J. A. Derbyshire, R. C. Boston, D. A. Bluemke, B. L. Gerber, J. L. Prince, and N. F. Osman, "Quantitative ischemia detection during cardiac magnetic resonance stress testing by use of FastHARP," Circulation, vol. 107, pp. 2025-30, 2003.

[43] C. Pislaru, C. J. Bruce, P. C. Anagnostopoulos, J. L. Allen, J. B. Seward, P. A. Pellikka, E. L. Ritman, and J. F. Greenleaf, "Ultrasound strain imaging of altered myocardial stiffness: stunned versus infarcted reperfused myocardium," Circulation, vol. 109, pp. 2905-10, 2004.

[44] K. U. Juergens, P. Reimer, T. P. Weber, B. Tombach, C. Bremer, B. Renger, H. V. Aken, and W. Heindel, "Cine and tagged magnetic resonance imaging in short-term stunned versus necrotic myocardium," International Journal of Cardiovascular Imaging, vol. 21, pp. 271-82, 2005.

[45] J. H. Omens, K. D. May, and A. D. McCulloch, "Transmural distribution of threedimensional strain in the isolated arrested canine left ventricle," American Journal of Physiology-Heart and Circulation Physiology, vol. 261, pp. H918-28, 1991.

[46] K. D. Costa, J. W. Holmes, and A. D. McCulloch, "Modeling cardiac mechanical properties in three dimensions," Philosophical Transactions of the Royal Society of London Series A, vol. 359, pp. 1233-50, 2001.

[47] A. J. Sinusas, X. Papdemetris, R. T. Constable, D. P. Dione, M. D. Slade, P. Shi, and J. S. Duncan, "Quantification of 3-D regional myocardial deformation: shape-based analysis of magnetic resonance images," American Journal of Physiology - Heart and Circulatory Physiology, vol. 281, pp. H698-H714, 2001. 
[48] T. P. Usyk, R. Mazhari, and A. D. McCulloch, "Effect of laminar orthotropic myofiber architecture on regional stress and strain in the canine left ventricle," Journal of Elasticity, vol. 61, pp. 143-164, 2000.

[49] D. H. Lin and F. C. Yin, "A multiaxial constitutive law for mammalian left ventricular myocardium in steady-state barium contracture or tetanus," J Biomech Eng, vol. 120, pp. 504-17, 1998.

[50] J. C. Walker, M. B. Ratcliffe, P. Zhang, A. W. Wallace, B. Fata, E. W. Hsu, D. Saloner, and J. M. Guccione, "MRI-based finite-element analysis of left ventricular aneurysm," Am J Physiol Heart Circ Physiol, vol. 289, pp. H692-700, 2005.

[51] R. B. King, J. B. Bassingthwaighte, J. R. Hales, and L. B. Rowell, "Stability of heterogeneity of myocardial blood flow in normal awake baboons," Circulation Research, vol. 57, pp. 285-95, 1985.

[52] J. B. Bassingthwaighte, M. A. Malone, T. C. Moffett, R. B. King, S. E. Little, J. M. Link, and K. A. Krohn, "Validity of microsphere depositions for regional myocardial flows," American Journal of Physiology, vol. 253, pp. H184-93, 1987.

[53] R. P. Shannon, K. Komamura, Y. T. Shen, S. P. Bishop, and S. F. Vatner, "Impaired regional subendocardial coronary flow reserve in conscious dogs with pacing-induced heart failure," American Journal of Physiology, vol. 265, pp. H801-9, 1993.

[54] M. S. O'Konski, F. C. White, J. Longhurst, D. Roth, and C. M. Bloor, "Ameroid constriction of the proximal left circumflex coronary artery in swine. A model of limited coronary collateral circulation," American Journal of Cardiovascular Pathology, vol. 1, pp. 69-77, 1987. 
[55] J. A. Fallavollita, "Spatial heterogeneity in fasting and insulin-stimulated (18)F-2deoxyglucose uptake in pigs with hibernating myocardium," Circulation, vol. 102, pp. 908-14, 2000.

[56] J. A. Fallavollita, C. Trojan, and J. M. Canty, Jr., "Transmural distribution of FDG uptake in stunned myocardium," American Journal of Physiology-Heart and Circulation Physiology, vol. 279, pp. H102-9, 2000.

[57] J. E. Lowe, R. G. Cummings, D. H. Adams, and E. A. Hull-Ryde, "Evidence that ischemic cell death begins in the subendocardium independent of variations in collateral flow or wall tension," Circulation, vol. 68, pp. 190-202, 1983.

[58] R. B. Jennings, C. Steenbergen, Jr., and K. A. Reimer, "Myocardial ischemia and reperfusion," Monographs in Pathology, vol. 37, pp. 47-80, 1995.

[59] H. B. Hillenbrand, R. J. Kim, M. A. Parker, D. S. Fieno, and R. M. Judd, "Early assessment of myocardial salvage by contrast-enhanced magnetic resonance imaging," Circulation, vol. 102, pp. 1678-83, 2000.

15 [60] R. J. Kim, E. Wu, A. Rafael, E. L. Chen, M. A. Parker, O. Simonetti, F. J. Klocke, R. O. Bonow, and R. M. Judd, "The use of contrast-enhanced magnetic resonance imaging to identify reversible myocardial dysfunction," New England Journal of Medicine, vol. 343, pp. 1445-53, 2000.

[61] A. D. McCulloch, D. Sung, J. M. Wilson, R. S. Pavelec, and J. H. Omens, "Flow20 function relations during graded coronary occlusions in the dog: effects of transmural location and segment orientation," Cardiovascular Research, vol. 37, pp. 636-45, 1998. 


\section{TABLES}

\begin{tabular}{ccccc}
\hline Heart & $\begin{array}{c}\text { LV Twist } \\
\text { (Base, } \\
\text { Apex) }\end{array}$ & $\begin{array}{c}\text { Longitudinal } \\
\text { Contraction of } \\
\text { LV Base }(\mathrm{mm})\end{array}$ & $\begin{array}{c}\text { LV Wall Thickening } \\
\text { (lateral, septal, } \\
\text { inferior, anterior) }(\%)\end{array}$ & $\begin{array}{c}\text { Ejection } \\
\text { Fraction (\%) }\end{array}$ \\
\hline 4D NCAT & $\left(5^{\circ},-13^{\circ}\right)$ & 14 & $(27,31,41,29)$ & 60 \\
Normal FE Model & $\left(6^{\circ},-11^{\circ}\right)$ & 10 & $(33,38,37,23)$ & 62 \\
Subendo FE Model & $\left(7^{\circ},-11^{\circ}\right)$ & 10 & $(31,36,37,12)$ & 58 \\
Trans FE Model & $\left(7^{\circ},-10^{\circ}\right)$ & 10 & $(29,33,37,3)$ & 55 \\
\hline
\end{tabular}

5 Table 1: Comparison of the LV Motion of the Normal Mechanical [FE] Model to that of the 4D NCAT Phantom indicate excellent agreement between the gross deformations predicted by the FE models and deformations of the NCAT phantom. The NCAT results are based on tagged MRI displacements for a normal human subject. The NCAT results are based on detailed measurements using tagged MRI analysis of the cardiac deformation for a normal 10 male subject.

\begin{tabular}{ccccccccc}
\hline $\begin{array}{c}\text { Strain } \\
\text { Component }\end{array}$ & \multicolumn{2}{c}{ FE Model } & \multicolumn{2}{c}{ Sinusas [47] } & \multicolumn{2}{c}{ Guccione [16] } & Omens [45] \\
& Endo & Epi & Endo & Epi & Endo & Epi & Endo & Epi \\
Circumferential & 0.29 & 0.07 & 0.15 & 0.07 & 0.15 & 0.09 & 0.22 & 0.05 \\
Radial & 0.33 & 0.15 & 0.25 & 0.15 & 0.34 & 0.19 & 0.18 & 0.12 \\
In-plane Shear & 0.01 & 0.01 & $<0.02$ & 0.06 & 0.01 & 0.03 & $0.02^{*}$
\end{tabular}

Table 2. Comparison of the FE strain predictions to values found in the literature. The forward FE results are in reasonable agreement with published experimental results. * Reported a mid-wall peak of 0.08 . 


\begin{tabular}{ccccc}
\hline Strain Component & \multicolumn{2}{c}{ FE Model } & \multicolumn{2}{c}{ Waldman Data ([41]) } \\
& Endo & Epi & Endo & Epi \\
\hline Circumferential (C) & 0.29 & 0.07 & 0.15 & 0.09 \\
Radial (R) & 0.33 & 0.15 & 0.34 & 0.19 \\
Longitudinal (L) & 0.09 & 0.10 & 0.07 & 0.05 \\
In-plane Shear (CR) & 0.01 & 0.01 & 0.06 & 0.01 \\
Circ./Long. Shear (CL) & 0.01 & 0.04 & 0.04 & 0.04 \\
Radial/Long. Shear (RL) & 0.08 & 0.01 & 0.08 & 0.00
\end{tabular}

Table 3. Comparison of the absolute values of the FE strain predictions to values found in the Waldman canine study [41]. The forward FE results are in excellent agreement with these 5 published experimental results for all of the strain values with the exception of the endocardial circumferential strain which the normal model prediction is higher than that measured in the Waldman study indicating a greater cirumferential strain gradient across the wall than that measured in the referenced study. 


\section{FIGURE CAPTIONS}

Fig. 1. (A) Anterior view of the 3D NCAT phantom showing detailed segmented organs from the Visible Human CT dataset. (B) A 3D image of the cardiac model of the NCAT phantom with the left anterior descending (LAD) and left circumflex (LCX) branches of the coronary artery superimposed on the outer surface of the heart model. The inner and outer surfaces and the motions of the left and right ventricles were extracted from gated tagged MR images of a normal male subject. Since the tagged MRI data did not cover the atria, the right and left atria models were created based on a separate set of MRI data from the University of Auckland [S. Thrupp, Cardiac MRI Anatomical Atlas, Available: www.scmr.org/education/atlas/intro/index.html]. This data was used to define the initial anatomy of the atria. The time curves for the cardiac volumes for a healthy male [M. Guyton, Textbook of Medical Physiology, Philadelphia: W.B. Saunders Co., 2000.] were used as a guide to scale the atria in 3D to fit the time-changing ventricular models. (C) A 3D image of the left ventricle model of the NCAT phantom showing the inner and outer surfaces, the LAD

15 and LCX branches of the coronary artery tree, a simulated atherosclerotic plaque and a highlighted anterior myocardial infarction.

Fig. 2. Left - finite element mesh for left ventricle model. Yellow elements surrounding the myocardium depict the tether mesh, which restricts rigid body motion. Right - Detail of the 20 LV mesh with the tether mesh removed.

Fig. 3. (A) Active contraction stress and (B) the left ventricular pressure load were used as inputs to the normal and pathological FE models. Simulations were performed from time 
zero, which represents beginning diastole, and continued through end-diastole $(425 \mathrm{msec})$ and end-systole $(650 \mathrm{msec})$. The active contraction stress $\left(T_{\max } C_{t}\right)$ was modified by the length dependent components of Equation (6) for each element in the model.

Fig. 4. (Left) Long-axis view of the node points defining the FE mesh for the LV at time 0. (Right) Short-axis view of the node points located at the base of the LV. The node points were arranged in a regular order, facilitating the definition of control points for an inner, outer, and five mid-ventricular NURBS surfaces.

Fig. 5. NURBS surfaces defined from the FE model developed for the normal heart. Three time points in the cardiac cycle are shown. Sets of time-position cubic NURBS curves derived from the FE analysis define the motion of the surfaces over the cardiac cycle. From the continuous time-position curves, an infinite number of time frames over the cardiac cycle can be produced using cubic spline interpolation.

Fig. 6: Views of the normal FE model at end-diastole (left column) and end-systole (right column). Base of LV (top), mid-ventricular cross-section (middle) and anterior view (bottom). Wall thickening and apical twist at end-systole can be seen in the figures on the right column as well as the movement of the base toward the apex.

Fig. 7: Mean transmural strain distributions of radial strain (solid line), circumferential strain (long dashed line), and fiber strain (dashed line with two dots) for the entire normal LV. The error bars indicate plus and minus one standard deviation of the corresponding mean. 
Negative values indicate compression and positive values indicate tension. Maximum circumferential and radial strain values occurred at the endocardium. All values are with respect to end-diastole.

5 Fig. 8. Comparison of strains predicted by FE models for the mid-ventricular cross-section. (A) Highlighted rectangular portion of the anterior LV indicates location of the ischemic region, while the cutting plane indicates location of short axis slices. Short axis cross-sections of the systolic strain distributions for the (B) normal, (C) subendocardial, and (D) transmural ischemic models. The top row indicates the radial strain results, the middle row depicts the circumferential strain results, and the bottom row is fiber strain. Strain values are referenced to the geometry at the beginning of diastole. The cross-sections are in radiological orientation with the anterior portion of the LV at the 12:00 position. The anterior wall of the LV as depicted in the normal model shows fiber and circumferential contraction as well as radial wall thickening. In the subendocardial model, the subendocardial region is undergoing

15 elongation in the fiber and circumferential directions and little change in wall thickness in the radial direction. In the transmural model, results show transmural elongation in the fiber and circumferential directions and wall thinning.

Fig. 9. Transmural strain distributions predicted by the three FE models. (A) fiber strain, (B) 20 circumferential strain and (C) radial strain, for the ischemic region shown in Figure 8A for the normal (solid line) tissue, subendocardial ischemic (long dashed line) tissue and transmural ischemic (short dashed line) tissue. The error bars indicate plus and minus one standard deviation of the corresponding mean value. Detailed analysis of the entire ischemic region 
indicates that the normal model shows fiber and circumferential contraction as well as radial wall thickening. The results for the subendocardial model indicate that the subendocardial region is undergoing elongation in the fiber and circumferential directions and little change in wall thickness in the radial direction while the epicardial region is undergoing fiber and

5 circumferential contraction. The transmural model results show transmural elongation in the fiber and circumferential directions and wall thinning.

Fig. 10. Short axis cross-sections of the systolic strain distributions for the (A) normal, (B) subendocardial, and (C) transmural ischemic FE models compared with the NCAT-based strain distributions for the cases of (D) $50 \%$ and (E) 100\% reduction in displacement in the transmural ischemic region. Top row indicates the radial strain results, the middle row depicts the circumferential strain results, and the bottom row is fiber strain. Strain values are referenced to the geometry at the beginning of diastole. Both of the NCAT-based transmural strain distributions show little in the way of strain changes. Strain values are referenced to 15 the geometry at the beginning of diastole.

Fig. 11. Transmural strain distributions predicted by the transmural FE model compared with the strain distribution from the NCAT ischemia definition. (A) fiber strain, (B) circumferential strain and (C) radial strain for the ischemic region shown in Figure 8A for the

20 FE based transmural ischemic (solid line) model, the 50\% reduction NCAT based ischemia results (long dashed line) and the fixed NCAT based ischemia (short dashed line) results. The fixed NCAT based transmural strain distribution shows little strain magnitude or variation over the entire thickness within the ischemic region. 
Fig. 12. Mid-ventricular simulated SPECT short axis images with a $20 \%$ reduction in perfusion, and a $50 \%$ reduction in perfusion. These NCAT simulation are based on the (row A) normal FE model, (row B) the subendocardial FE model and (row C) the transmural FE 5 model. The subendocardial models are difficult to distinguish from the normal simulations for both of these perfusion cases. The arrow indicates the site of the ischemic region in the transmural simulation with a $50 \%$ reduction in perfusion.

Fig. 13. Intensity profiles (top) across the anterior region of the LV taken from short-axis images (bottom) indicate that the transmural defect based on the FE model shows the greatest reduction in intensity with respect to the normal. This is only due to the wall thinning which resulted from the FE model simulation. The other cases show no wall thinning. All of the simulations were defined having normal perfusion so the changes in intensity across the wall are due to geometric affects only. The labels "NCAT Trans. 50\%" and "NCAT Trans. 100\%" 15 refers to the simple definition of ischemia implemented in the 4D NCAT where the wall motion is reduced by $50 \%$ and $100 \%$ respectively. 


\section{FIGURES}

Figure 1.

(column width)
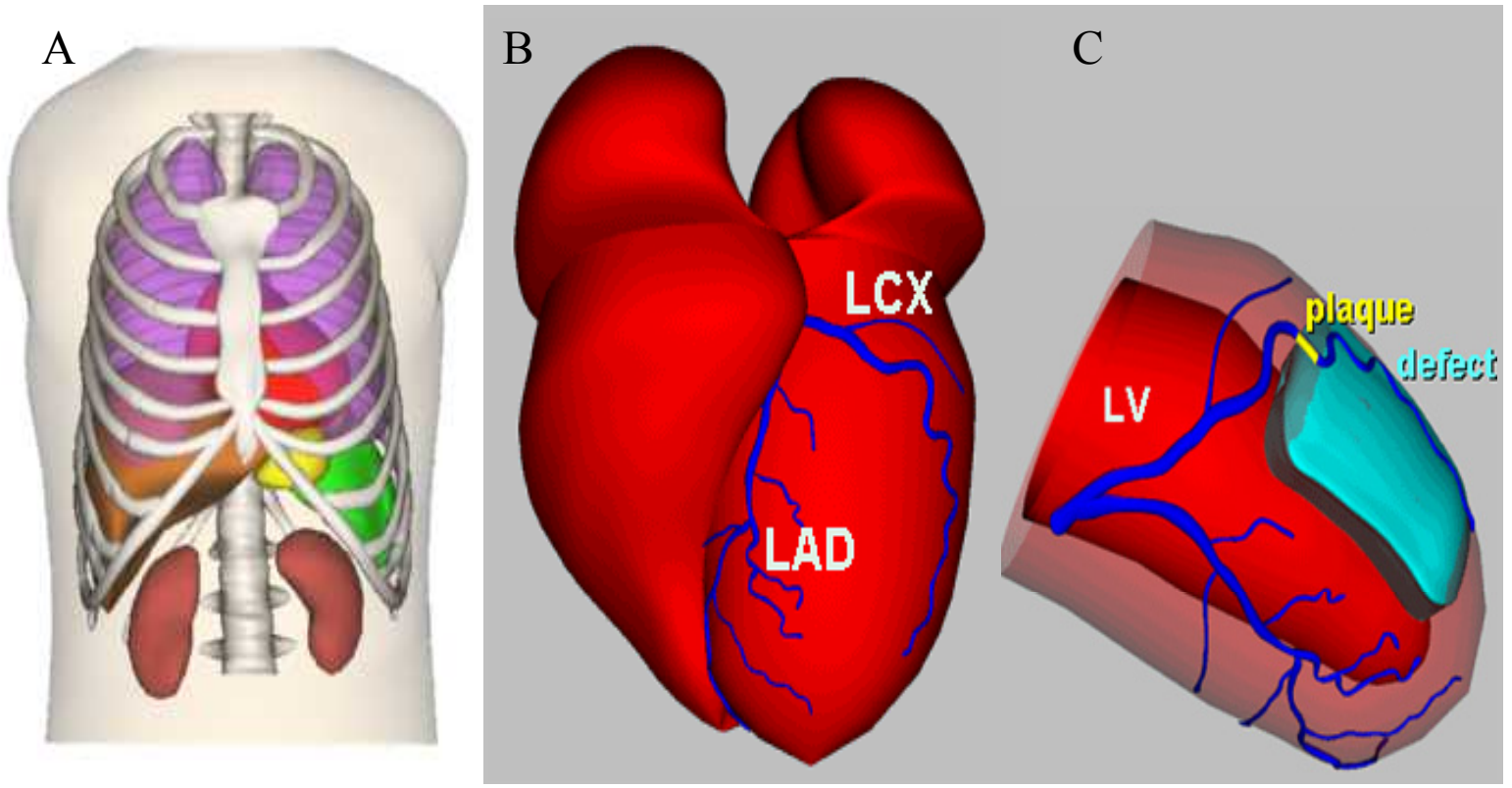

5 
Figure 2.

(column width)

5
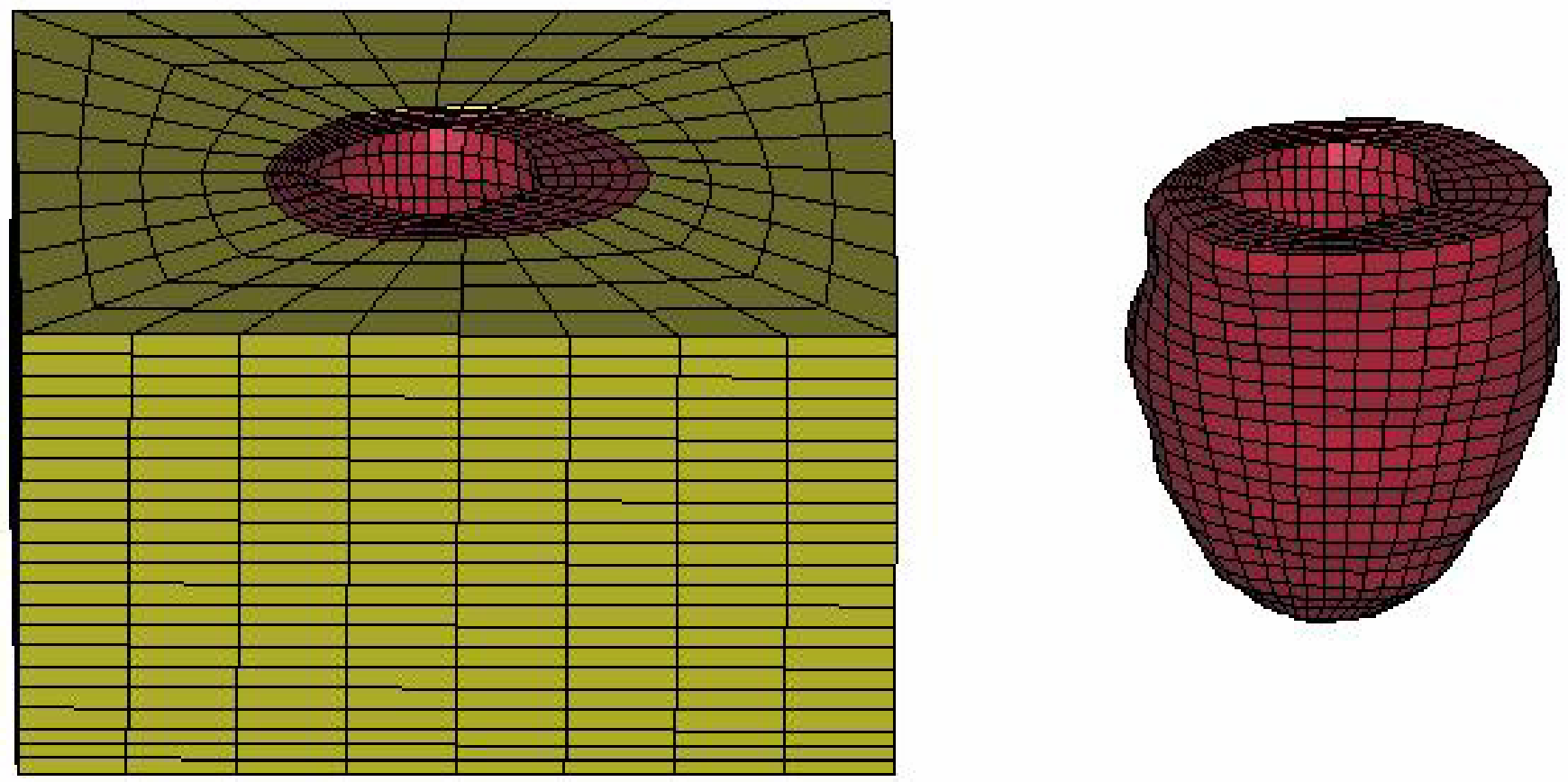
Figure 3

(column width)
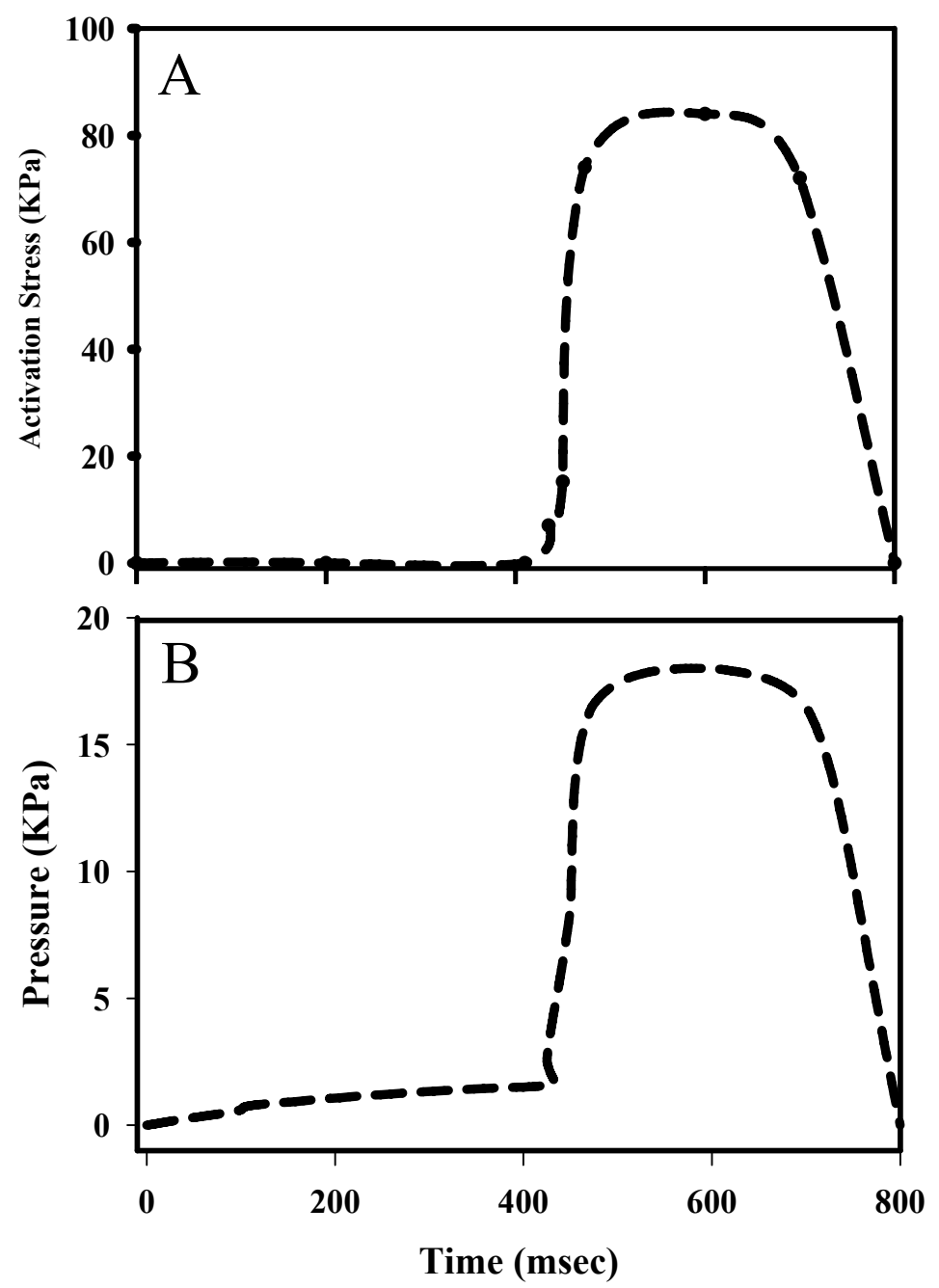
Veress et al.

Figure 4.

(column width)

5
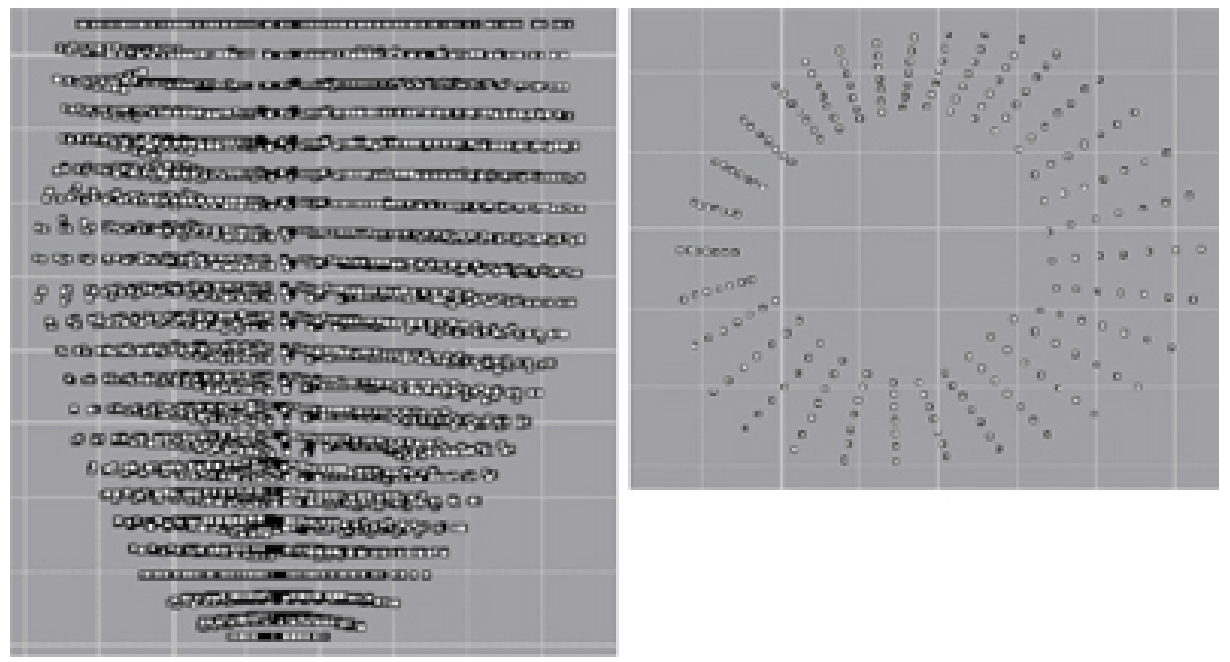
Figure 5

(page width)
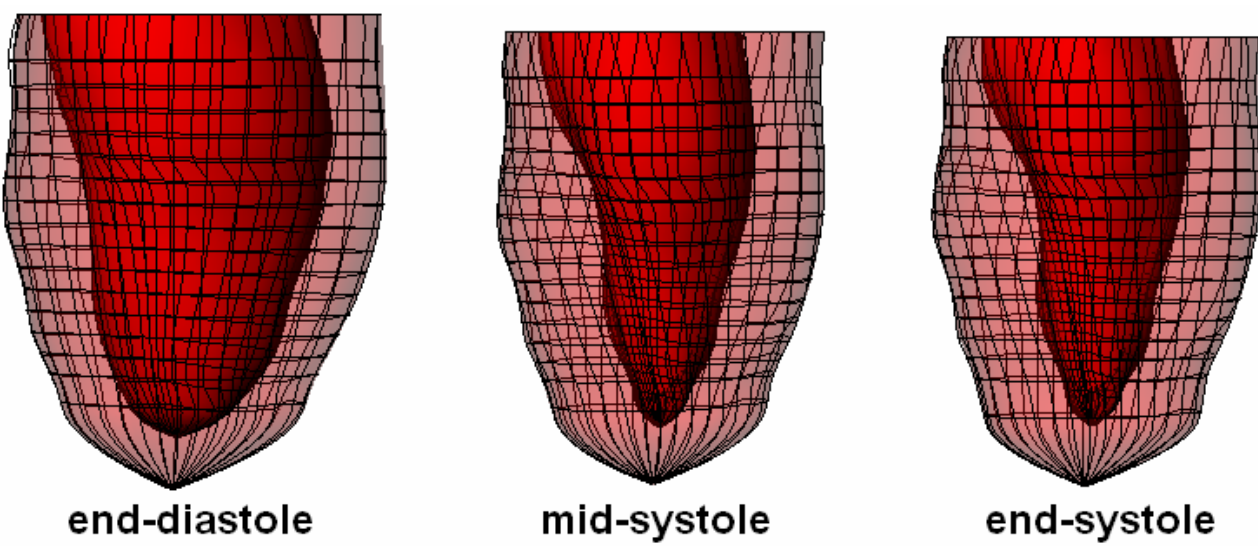
Figure 6.

(column width)
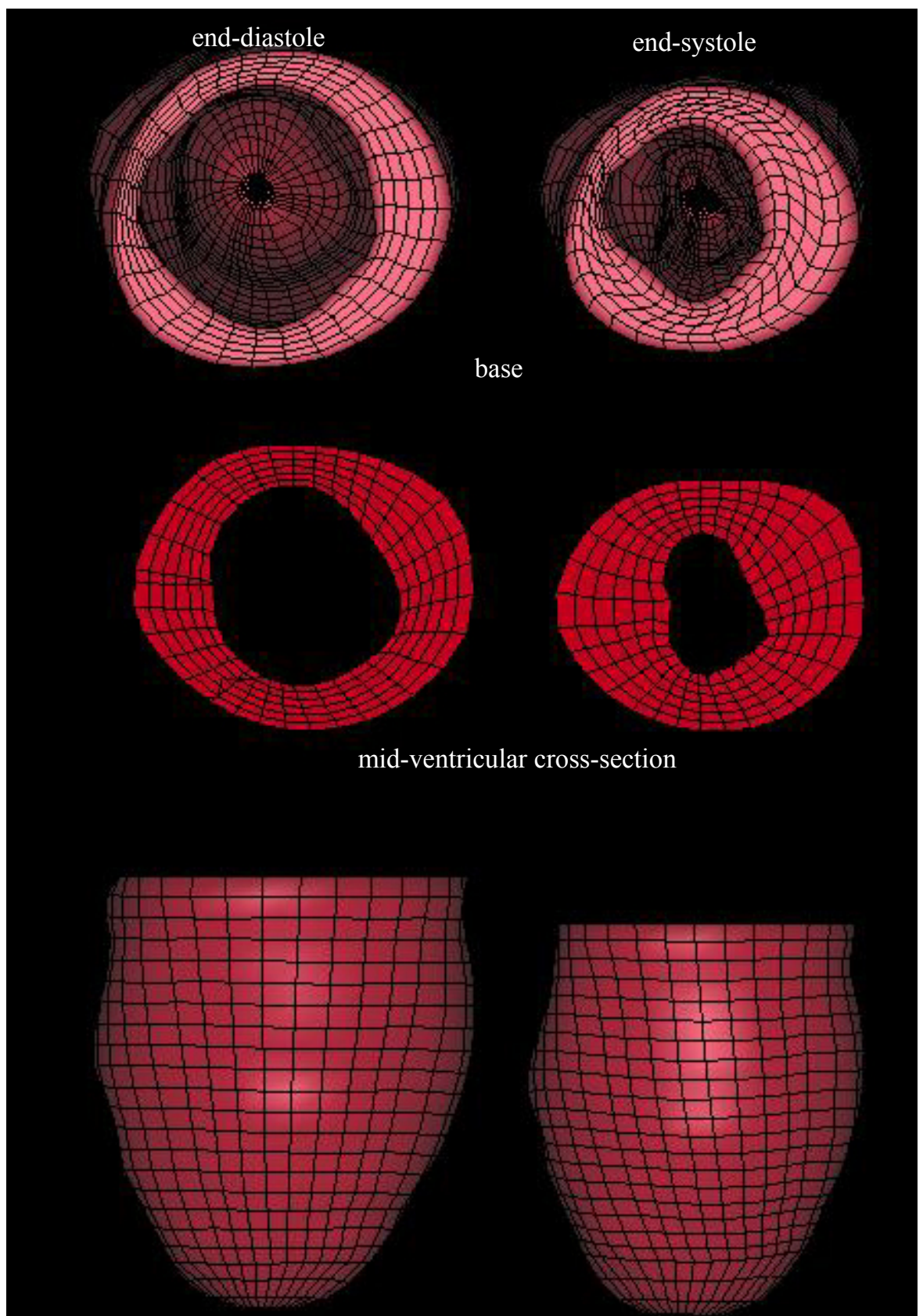

anterior view 
Figure 7.

(column width)

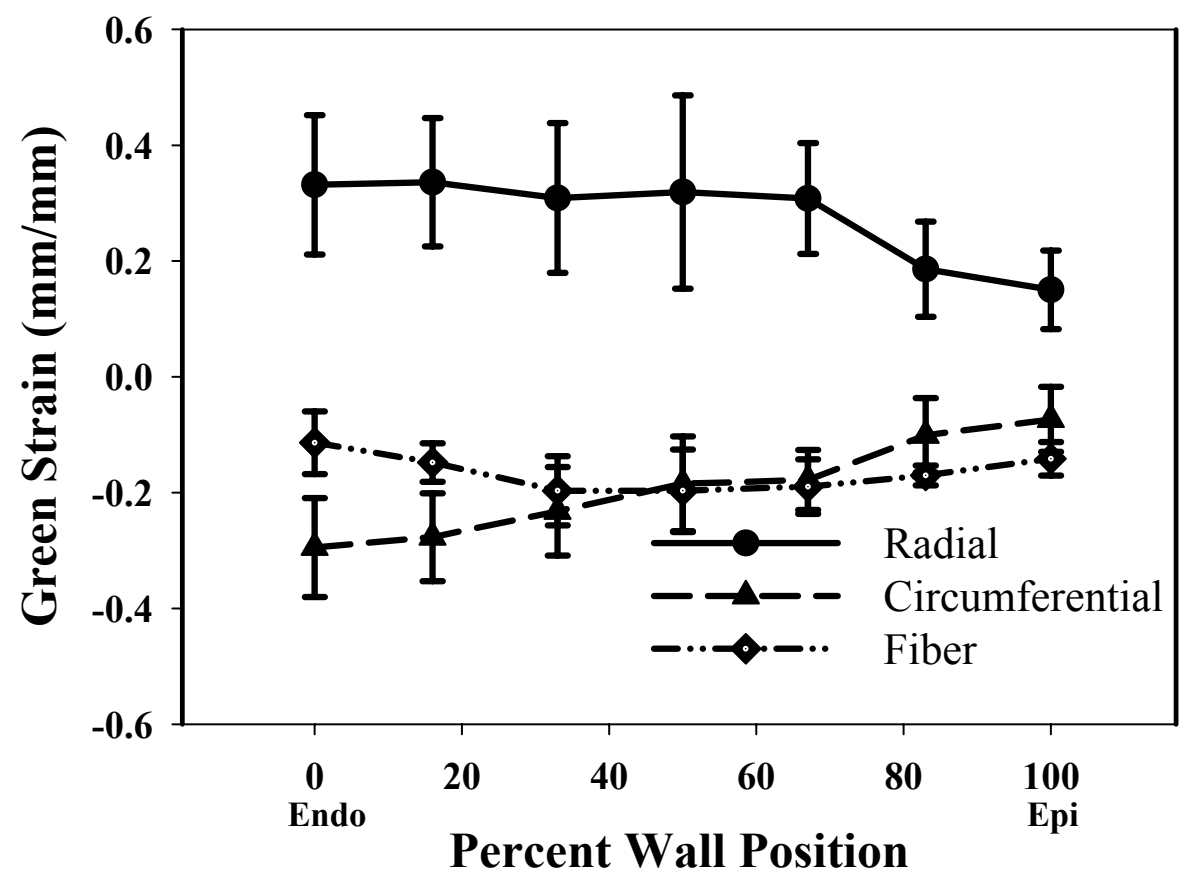


Figure 8 .

(page width)

5

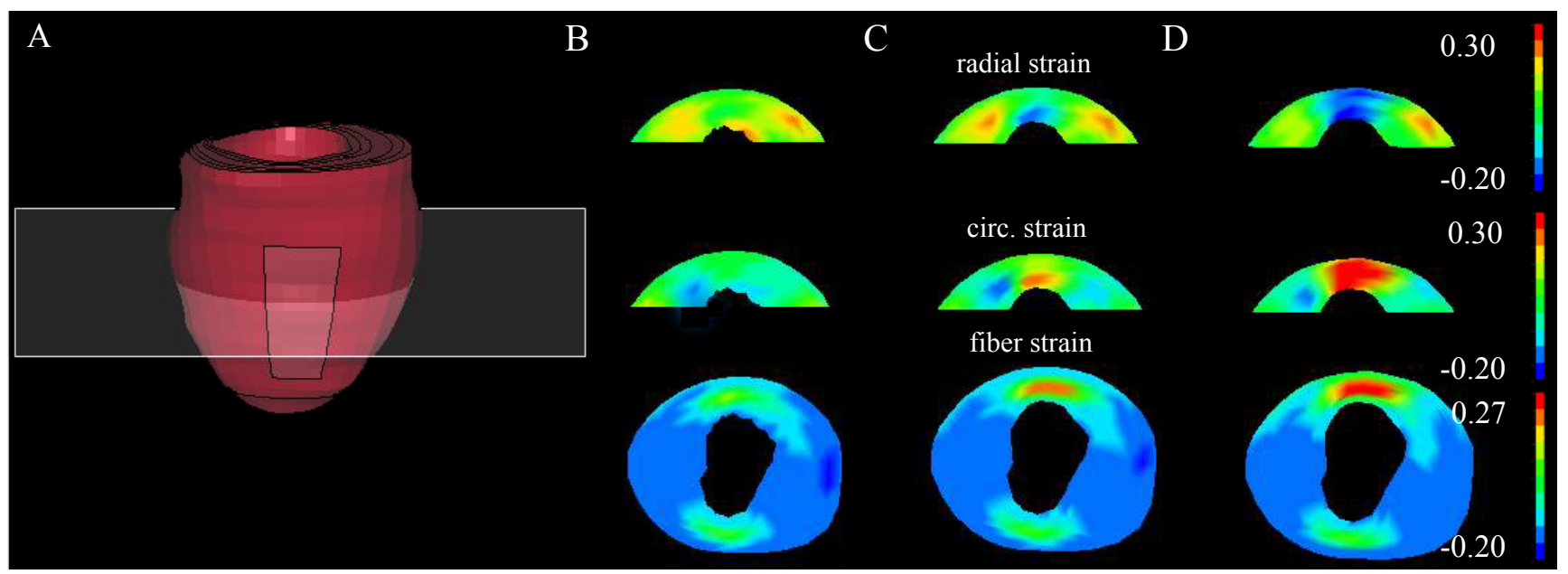


Figure 9

(column width)
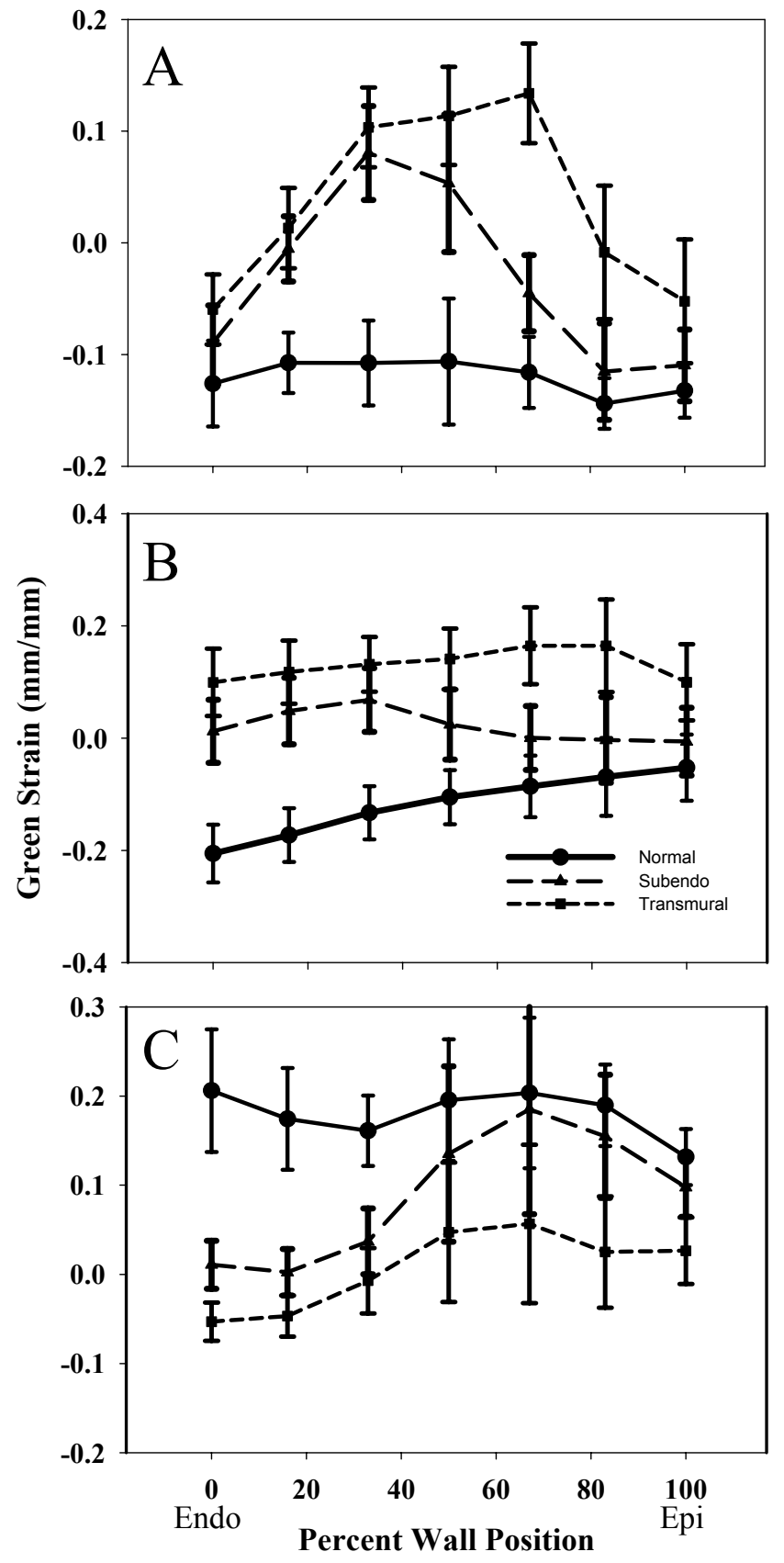
Figure 10

(page width)

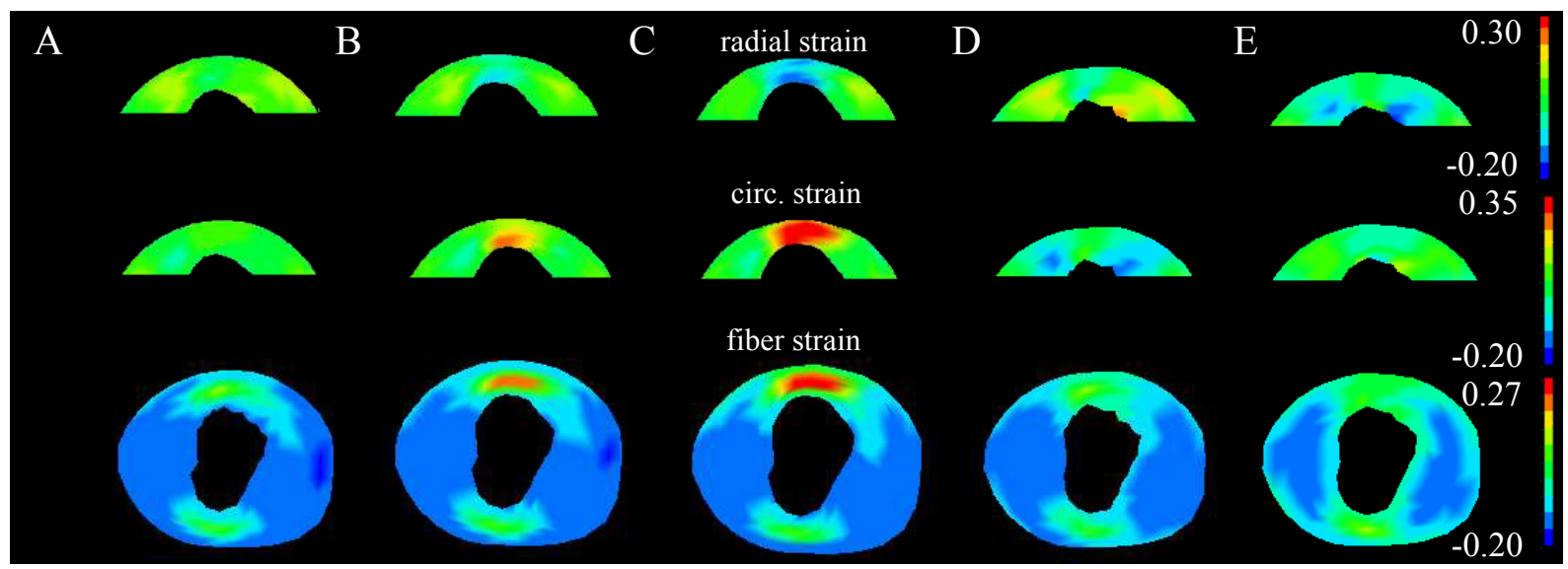


Figure 11

(column width)
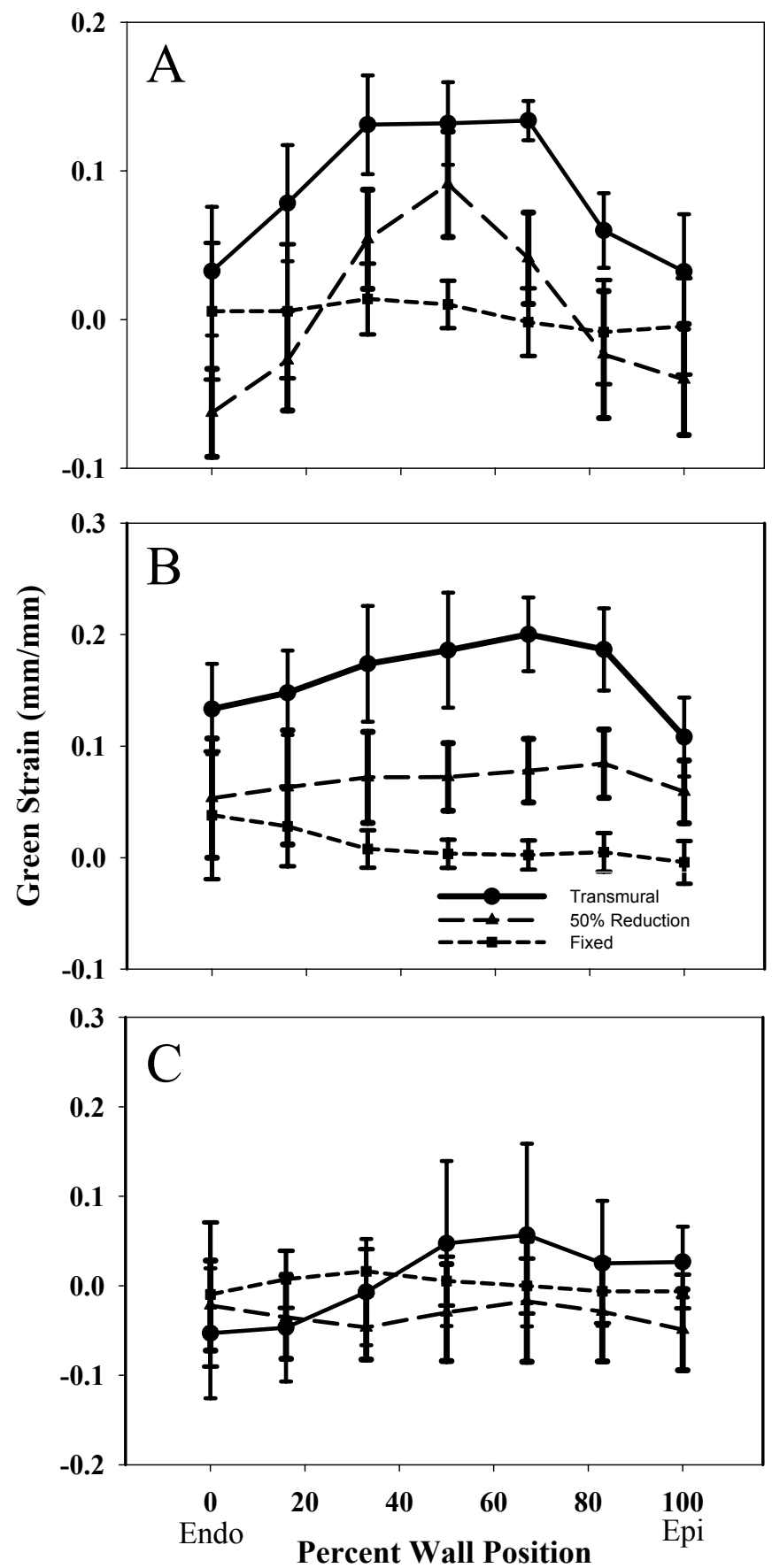
Figure 12

(column width)

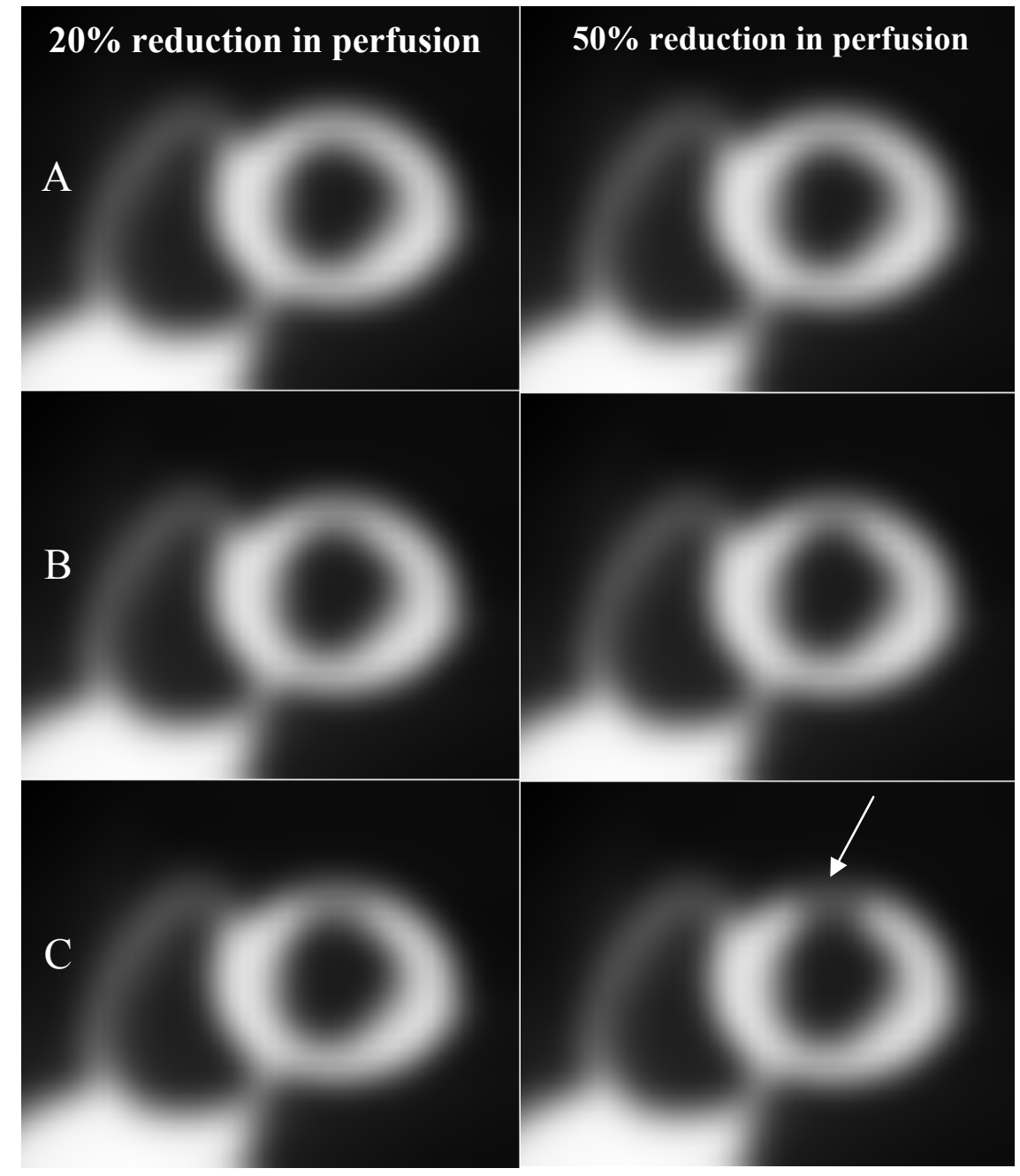


Figure 13

(page width)

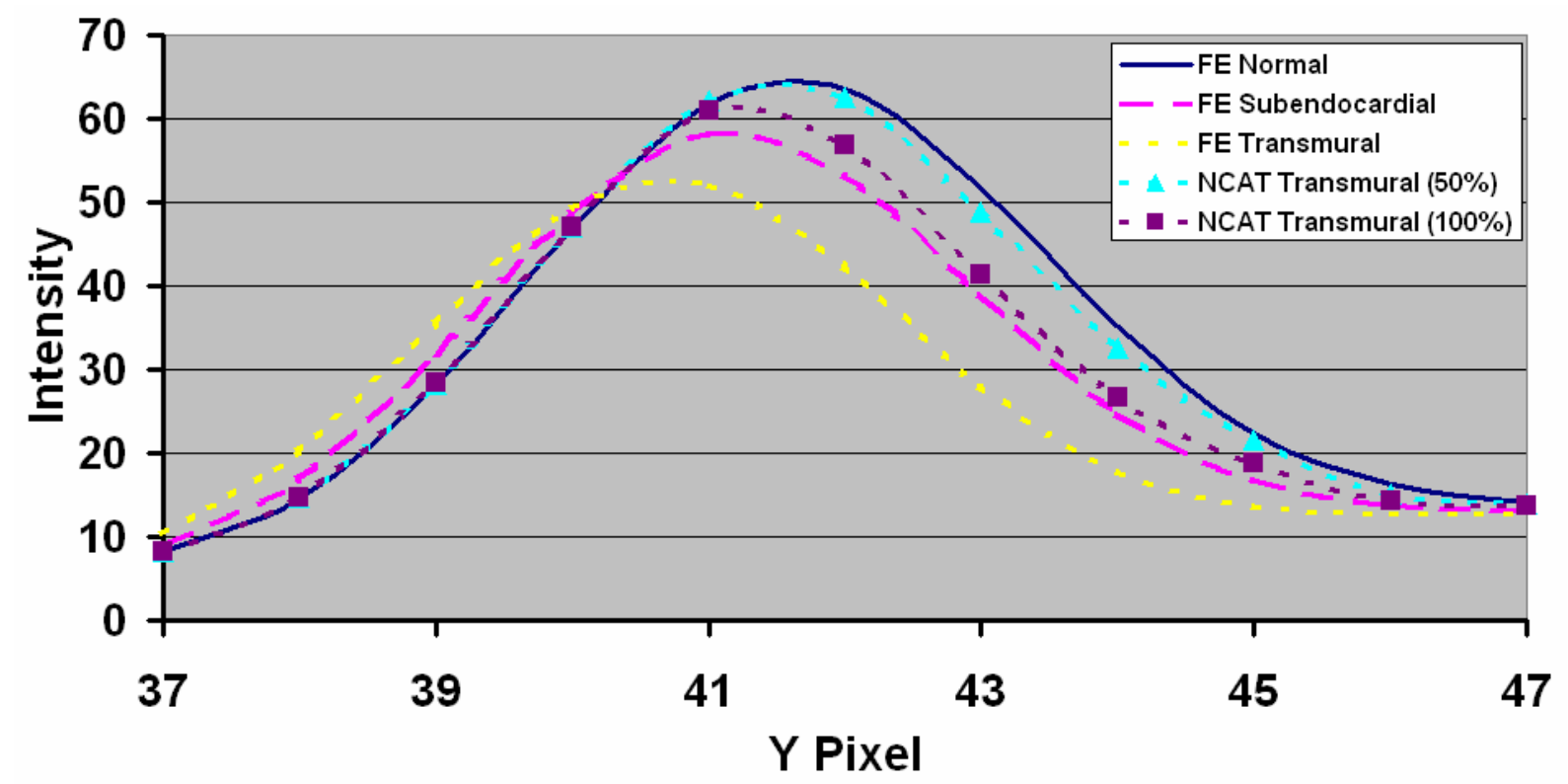

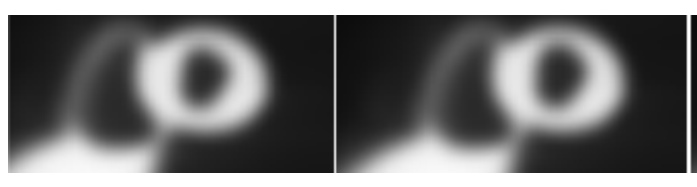

FE normal
FE Subendo

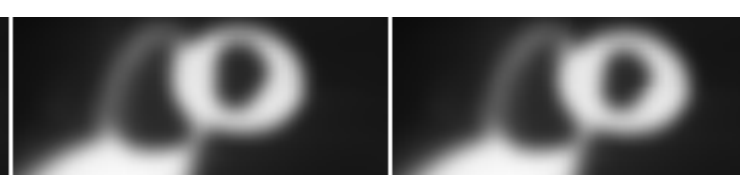

FE Transmural

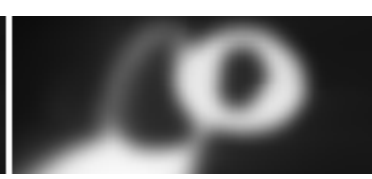

NCAT Trans $(50 \%)$

NCAT Trans (100\%) 